\title{
Pion production in nonequilibrium chiral perturbation theory
}

\author{
A. Gómez Nicola* \\ Departamento de Física Teórica II, Universidad Complutense, 28040 Madrid, Spain
}

(Received 15 March 2001; published 12 June 2001)

\begin{abstract}
We apply the formalism of chiral perturbation theory out of thermal equilibrium to describe explosive production of pions via the parametric resonance mechanism. To lowest order the Lagrangian is that of the nonlinear sigma model where the pion decay constant becomes a time-dependent function. This model allows for a consistent nonequilibrium formulation within the framework of the closed time path method, where one-loop effects can be systematically accounted for and renormalized. We work in the narrow resonance regime where there is only one resonant band. The pion distribution function is peaked around the resonant band where the number of pions grow exponentially in time. The present approach is limited to remain below the back-reaction time, although it accounts for nearly all the pion production during the typical plasma lifetime. Our results agree with the analysis performed in the $O(4)$ model. The space and time components $f_{\pi}^{s, t}(t)$ are also analyzed. To one loop $f_{\pi}^{s} \neq f_{\pi}^{t}$ unlike the equilibrium case and their final central values are lower than the initial ones. This effect can be interpreted in terms of a reheating of the plasma.
\end{abstract}

DOI: 10.1103/PhysRevD.64.016011 PACS number(s): 11.10.Wx, 11.30.Rd, 12.39.Fe, 25.75.-q

\section{INTRODUCTION}

Nonequilibrium quantum field theory has attracted considerable attention over the past decade, partially motivated by the experiments on relativistic heavy ion collisions. The ultimate goal of such experiments is to describe the properties of the QCD phase diagram and the quark-gluon plasma (QGP) from the observation of the final hadronic spectra. The Relativistic Heavy Ion Collider (RHIC) at BNL, which is already running, will reach energy densities high enough to confirm the very promising results obtained at the CERN Super Proton Synchrotron (SPS) during the last few years, indicating the existence of the QGP [1]. For a recent update of both theoretical and experimental results in this area see [2]. A lot of theoretical effort has been put into trying to understand the various properties of the QCD phase diagram in different ranges of temperatures and densities in thermal equilibrium. In addition, there are many aspects of the nonequilibrium behavior of the plasma which are not fully understood. In the standard picture of the collision $[3,4]$ the plasma formed in the central rapidity region cools down very rapidly, reaching approximate local thermal equilibrium. During the subsequent expansion, the temperature scales of chiral phase transition and deconfinement are crossed and hadrons are produced. In this regime, observables depend only on proper time approximately, in the central region. The expansion goes on until the final freeze-out of hadrons. The typical plasma lifetime during which nonequilibrium effects are important and most of the final hadrons are produced is about $10 \mathrm{fm} / c$.

A possible scenario to explain the observed final hadron distributions is that where strong fluctuations of the pion field are formed during the chiral phase transition, giving rise to the so called disoriented chiral condensates (DCC) [5]. These were suggested originally as misaligned vacuum regions where the chiral field is pointing out in a different

*Email address: gomez@eucmax.sim.ucm.es direction in isospin space from that where the vacuum expectation value of the pion field vanishes. Ideally, one would have misaligned regions of observable size, with only neutral (or only charged) pions. If such regions were formed, one would observe large clusters of pions emitted coherently from the plasma as the pion field relaxes to the normal vacuum [6]. This kind of behavior is indeed observed in Centauro events in cosmic ray experiments [7]. A clear signal for DCC formation has not yet been observed in RHIC experiments [8]. However, it seems that one has to measure higher order pion correlation functions in order to identify a pure DCC signal which is not masked by other effects $[9,10]$ so that the search will continue at BNL. Other observable consequences of DCC-like configurations would be an enhancement of dilepton and photon production $[11,12]$ and a modification of the effective $\pi^{0} \rightarrow \gamma \gamma$ vertex [13]. In any case, pion production within the energy scales of the chiral symmetry provides a natural framework for hadronization [14].

Thus, one should be able to describe nonequilibrium phenomena such as large pion production, from the microscopic theory governing the relevant degrees of freedom. At the energy scales where the chiral symmetry plays a predominant role (below $1 \mathrm{GeV}$ ) QCD is nonperturbative and one has to use an effective Lagrangian which describes satisfactorily the microscopic meson dynamics. Such a theory must incorporate the QCD symmetries and the chiral spontaneous symmetry breaking (SSB) pattern. In this picture the NambuGoldstone bosons (NGB) are the lightest mesons $(\pi, K, \eta)$ and the masses of the light quarks are meant to be treated perturbatively. One possible choice is simply the $O(N)$ model where the fundamental fields are $N-1$ pions and the $\sigma$, and the potential has the typical SSB shape. However, one should bear in mind that this model becomes nonperturbative in the coupling constant at low energies so that it is imperative to perform alternative expansions such as large $N$. On the other hand, the $O(N)$ model shares the QCD chiral symmetry breaking pattern only for $N=4$, so that it is not able to incorporate kaons and etas. 
An alternative approach is an effective theory built as an infinite sum of terms with increasing number of derivatives, only in terms of the NGB fields. The nonlinear sigma model (NLSM) is the lowest order action one can write down in this expansion. Higher order corrections come both from NGB loops and higher order Lagrangians and can be renormalized order by order in energies, yielding finite predictions for the meson observables. The unknown coefficients, which encode all the information on the underlying theory, absorb the loop infinities and their finite part can be fitted to experiment. This framework constitutes the so called chiral perturbation theory (ChPT) $[15,16]$ which provides a well-defined perturbative expansion in terms of $p / \Lambda_{\chi}$, where $p$ stands generically for any meson energy scale of the theory (masses, external momenta, temperature and so on) and the chiral scale $\Lambda_{\chi} \simeq 1.2 \mathrm{GeV}$ (see $[17,18]$ for a review). One of the many advantages of this scheme is that it can be extended from the $S U(2)$ chiral symmetry (only $\pi$ fields) to $S U(3)$ with $K$ and $\eta$. The ChPT formalism has also been applied in thermal equilibrium to analyze various properties of the low temperature meson gas [19-21].

In the context of nonequilibrium chiral dynamics, two possible scenarios for pion production and DCC formation have been proposed: the first one takes place in the early stages of the plasma evolution. Roughly speaking, after a very rapid cooling the chiral field is at the top of the classical potential in the chirally broken phase. As the field rolls down, long wavelength modes grow exponentially (spinodal instabilities) and this behavior is responsible for the enhancement of DCC's. There have been several approaches in the literature to implement this idea in the $O(4)$ model $[6,22,23]$. Typically, the pion distribution function is peaked at low momenta, being different from a thermal distribution [23] whereas the pion densities and DCC sizes predicted are around $n_{\pi} \simeq 0.2 \mathrm{fm}^{-3}$ and $1.5-2 \mathrm{fm}$ respectively. The second suggestion is based on the parametric resonance mechanism [14] and inherits the idea from inflationary reheating [24]. The analysis in the spinodal regime shows that the time it takes for the field to roll down to the bottom is very short compared with the total plasma lifetime. Thus, in the parametric resonance approach, the $\sigma$ field is oscillating around the minimum of the potential in a later stage of the plasma evolution. Those oscillations transfer energy to the pion modes, giving rise to pion solutions exponentially growing in time via parametric resonance. Typically, the unstable modes develop in bands in momentum space and the more important resonance band is centered at $k \simeq m_{\sigma} / 2[9,25]$. The DCC sizes in this approach can be as large as $5 \mathrm{fm}$ [26] and recent calculations show that strong charge-neutral correlations in parametric resonance can be used to identify a pure DCC signal [9]. Furthermore, the reheating process yields predictions for the final hadronization temperature compatible with the observations [14]. One must stress that both approaches are complementary and in fact the initial conditions needed for the parametric resonance correspond to the final stage of the rolling down solution. A very detailed analysis of both regimes in the context of the $O(N)$ model can be found in [27].
The purpose of this work is to explore pion production in parametric resonance within the ChPT framework, as a complementary analysis to the $O(4)$ model. We will show that within this formalism one can also describe regions where the number of pions and the pion correlator grow exponentially. The main advantages of the ChPT approach are that one can follow a consistent perturbative treatment which is renormalizable order by order and that it can be extended to three flavors. Besides, one is dealing only with NGB fields, although we will show how the pion production can be understood in terms of the $\sigma$ field evolution in the $O(4)$ model. This method is best suited for the stage of the plasma expansion where the system is well into the broken phase of the chiral symmetry. This is precisely the regime where parametric resonance takes place.

We will build on a previous work [28], where we have analyzed the extension of ChPT out of thermal equilibrium. In that work it has been shown that the power counting and renormalization program can be consistently implemented also at nonequilibrium. In turn, the present analysis will provide a particular example where it will be shown explicitly how the chiral power counting and renormalization program work, yielding predictions for physical observables. The key idea is to make use of the derivative expansion consistently implemented in ChPT in order to study the system not far from equilibrium. For that purpose, the nonequilibrium dynamics is encoded effectively in the parameters of the model. To leading order and assuming a spatially homogeneous system, we let the pion decay constant be time dependent. This function acts as an external force on the pion degrees of freedom. It is important to bear in mind that a self-consistent treatment should amount to incorporate the full hydrodynamics of both the fluid and pion modes [29]. In the present approach we will concentrate only on the influence of the expansion on the meson dynamics. This is a similar situation as considering a quantum field theory (QFT) in the presence of an external curved background space-time [30]. In that case, it makes sense under certain conditions to ignore the back-reaction effect of the matter fields in the metric. Similarly, we will see that for the time scales relevant for pion production it is reasonable to ignore those effects and treat the influence of the expanding plasma as external.

The paper is organized as follows: In Sec. II we will review the nonequilibrium $\mathrm{ChPT}$ and its relationship to curved space-time QFT, which will be crucial in what follows. The parametric resonance approach in its simpler version will be discussed in Sec. III, while Secs. IV and V will be devoted to analyze the effects of parametric resonance in two different observables: the pion decay functions and the pion number respectively. The latter is the most relevant observable as far as pion production is concerned whereas the former will allow us to estimate the time scale when the back-reaction effects become important as well as the final temperature by that time. In both cases we will calculate up to one-loop in ChPT, paying particular attention to renormalization. In addition, in Sec. $\mathrm{V}$ the definition of particle number we will use and its relationship with the energymomentum tensor are discussed. Our conclusions are summarized in Sec. VI. We have included two appendices 


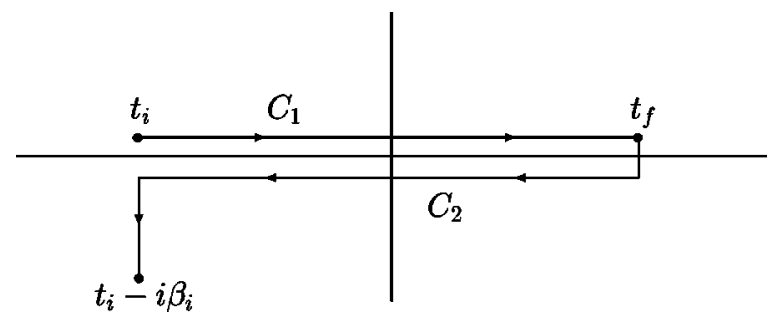

FIG. 1. The contour $C$ in complex time $t$. The lines $C_{1}$ and $C_{2}$ run between $t_{i}+i \epsilon$ and $t_{f}+i \epsilon$ and $t_{f}-i \epsilon$ and $t_{i}-i \epsilon$ respectively, with $\epsilon \rightarrow 0^{+}$.

collecting some useful results about the Mathieu equation and curved space-time QFT respectively.

\section{NONEQUILIBRIUM CHIRAL PERTURBATION THEORY}

\section{A. The NLSM out of thermal equilibrium}

The chiral Lagrangian to lowest order is the nonlinear sigma model (NLSM), which contains two derivatives of the fields. In the chiral limit, where the mass of the light quarks is set to zero, the NLSM only contains one energy parameter $f$. To lowest order, $f$ is nothing but the pion decay constant $f=f_{\pi} \simeq 93 \mathrm{MeV}\left(f \neq f_{\pi}\right.$ to higher orders). Consistently, our nonequilibrium model will be the NLSM where $f$ is replaced by a time-dependent function $f(t)$. This function acts as an external field encoding the time evolution of the system as, for instance, the expansion of the plasma formed after a RHIC in proper time. As for the initial conditions we will assume that the system is in thermal equilibrium before some initial time ( $t=0$ for convenience) at the temperature $T_{i}$ $=\beta_{i}^{-1}$. This is an important simplification from the point of view of the nonequilibrium path-integral formulation. In fact, one can formulate the generating functional for the real-time Green functions by extending the time arguments to the contour $C$ in the complex plane shown in Fig. 1. This is an extension of the closed time path technique [31] for nonequilibrium field theory, where the inclusion of the imaginarytime leg is a consequence of the choice of equilibrium initial conditions [32]. Thus, $f(t \leqslant 0)=f$, and the function $f(t)$ represents an external force switched on at $t=0$ and driving the system out of equilibrium. Note that we choose that departure to be instantaneous and then $f(t)$ cannot be analytical at $t=0$. Nevertheless, it is natural to expect that these restrictions on the initial conditions do not influence very much the physical results for longer times, especially if the system is not far from equilibrium.

Thus, our starting point will be the following nonequilibrium NLSM action:

$$
S_{2}[U]=\int_{C} d^{4} x \frac{f^{2}(t)}{4} \operatorname{tr} \partial_{\mu} U^{\dagger}(\vec{x}, t) \partial^{\mu} U(\vec{x}, t)
$$

where $\int_{C} d^{4} x=\int_{C} d t \int d^{3} \vec{x}$. We will restrict here to the case of two light quark flavors (i.e, the NGB are only the pions) and hence $U(x) \in S U(2)$. In addition, we will be interested only in the chiral limit i.e., massless pions. Thus, we are not in- cluding any explicit symmetry-breaking term in the action. Note that the action (1) is chiral invariant $\left[U \rightarrow L U R^{\dagger}\right.$ where $L, R$ are constant $S U(2)$ matrices] by construction, which will play an important role in what follows. As it is customary, $U(x)$ is parametrized in terms of the pion fields $\pi^{a}(\vec{x}, t)$ as

$$
U(\vec{x}, t)=\frac{1}{f(t)}\left\{\left[f^{2}(t)-\pi^{2}(\vec{x}, t)\right]^{1 / 2} I+i \tau_{a} \pi^{a}(\vec{x}, t)\right\}
$$

where $I$ is the $2 \times 2$ identity matrix, $\tau_{a}$ are the Pauli matrices, $\pi^{2}=\pi^{a} \pi_{a}$ and $\pi^{a}\left(t_{i}-i \beta_{i}\right)=\pi^{a}\left(t_{i}\right)$ is the equilibrium [Kubo-Martin-Schwinger (KMS)] boundary condition, with $t_{i}<0$. As we will show below, the physics does not depend on the choice of $t_{i}$.

It is useful to recall that the NLSM can be viewed to lowest order as an $O(4)$ model subject to the constraint $\sigma^{2}$ $+\pi^{2}=f^{2}$. The same applies to the nonequilibrium case and therefore we can think of $f(t)$ as the lowest order expectation value of the $\sigma$ field in the $O(4)$ model, which has not reached its equilibrium value yet.

With the NLSM one can predict the very low energy behavior of pion observables such as the pion decay constant or the pion scattering amplitude, which agree with the current algebra predictions. However, to go beyond the lowest order, one has to consider pion loops. Thanks to Weinberg power counting theorem [15] we know that the loop diagrams are of the same order as Lagrangians with more derivatives of the pion fields. In fact, it can be shown explicitly that the (undetermined) coefficients of such Lagrangians absorb all possible UV divergences coming from the loops and hence one gets finite and scale-independent predictions for the pion observables.

In the present case, we need an extra ingredient to the power counting, namely, the time derivatives of the function $f(t)$. We will take

$$
\frac{\dot{f}(t)}{f(t)} \simeq \mathcal{O}(p), \quad \frac{\ddot{f}(t)}{f(t)}, \frac{[\dot{f}(t)]^{2}}{f^{2}(t)} \simeq \mathcal{O}\left(p^{2}\right)
$$

and so on. In this sense, one remains close to equilibrium. The rest of the power counting is the usual one, i.e., every derivative of the pion field is $\mathcal{O}(p)$ and every pion loop introduces an extra $\mathcal{O}\left(p^{2}\right)$. Other than being subject to the conditions (2), we will let $f(t)$ be arbitrary. However, in this work we shall discuss how $f(t)$ can be chosen consistently with physical results such as pion production.

Let us now expand the NLSM action to lowest order in the pion fields. Using the boundary conditions, we can integrate by parts and write, to second order in the pion fields,

$$
S_{2}[\pi]=-\frac{1}{2} \int_{C} d^{4} x \pi^{a}(\vec{x}, t)\left[\square+m^{2}(t)\right] \pi^{a}(\vec{x}, t)+\cdots
$$

where $m^{2}(t)=-\ddot{f}(t) / f(t)$. That is, the model accommodates a time-dependent pion mass term, without breaking explicitly the chiral symmetry (unlike a physical pion mass term). This 
is an important feature of this model, since it suggests an interesting connection with a QFT in the presence of an external curved space-time background. We will discuss this point in detail in Sec. II C. Before that, let us analyze the main properties of the leading order two-point function (the propagator) arising from the above NLSM action.

\section{B. The leading order propagator}

The two-pion correlation function is $i G^{a b}\left(x, x^{\prime}\right)$ $=\left\langle T_{C} \pi^{a}(\vec{x}, t) \pi^{b}\left(\vec{x}^{\prime}, t^{\prime}\right)\right\rangle$, where $T_{C}$ indicates time ordering along the contour $C$. Note that, from isospin invariance, we can write just $G^{a b}\left(x, x^{\prime}\right)=\delta^{a b} G\left(x, x^{\prime}\right)$. Besides, by spatial translation invariance the two-point function depends only on $\vec{x}-\vec{x}^{\prime}$. This is not true for the time coordinates due to the nonequilibrium time dependence in the Lagrangian.

There are four different types of propagator depending on the relative position of $t$ and $t^{\prime}$, namely, $G^{11}, G^{12}$ and so on [4]. Instead of writing all the combinations explicitly, we shall keep the condensed notation of time ordering with $T_{C}$ defining the natural extensions $\theta_{C}, \delta_{C}$ and so on. Thus, from Eq. (3), the leading order $G_{0}\left(x, x^{\prime}\right)$ is a solution of the differential equation

$$
\left\{\partial_{t}^{2}+k^{2}+m^{2}(t)\right\} G_{0}\left(t, t^{\prime}, k\right)=-\delta_{C}\left(t-t^{\prime}\right)
$$

where $G_{0}\left(t, t^{\prime}, k\right)$ is the Fourier transform in the space coordinates only and $k^{2}=|\vec{k}|^{2}$.

As for the boundary conditions, thermal equilibrium for $t<0$ means that we have to impose KMS boundary conditions at the imaginary-time leg in Fig. 1 [4]. That is, defining

$$
G\left(t, t^{\prime}, k\right)=G^{>}\left(t, t^{\prime}, k\right) \theta_{C}\left(t-t^{\prime}\right)+G^{<}\left(t, t^{\prime}, k\right) \theta_{C}\left(t^{\prime}-t\right),
$$

the KMS boundary conditions read

$$
G_{0}^{>}\left(t_{i}-i \beta_{i}, t^{\prime}, k\right)=G_{0}^{<}\left(t_{i}, t^{\prime}, k\right) .
$$

The general solution to the differential equation (4) with the boundary conditions (6) can be constructed for all the branches of the contour in terms of two particular solutions $h_{1,2}(t, k)$ to the homogeneous equation [33]:

$$
\left\{\partial_{t}^{2}+k^{2}+m^{2}(t)\right\} h_{i}(t, k)=0, \quad i=1,2,
$$

such that their Wronskian

$$
W(t, k) \equiv \dot{h}_{1}(t, k) h_{2}(t, k)-h_{1}(t, k) \dot{h}_{2}(t, k) \neq 0 .
$$

It is important to remark that the general solution $G_{0}\left(t, t^{\prime}, k\right)$ must be continuous and differentiable in the time coordinates so that it is uniquely defined. Thus, in our case we demand that $h_{i}(t, k)$ for $t>0$ and their first derivative match the equilibrium solutions at $t=0$. Note that, since Eq. (7) does not contain $\dot{h}$ terms, one can have solutions $h_{i}(t, k)$ which are continuous and differentiable at $t=0$ even though the function $m^{2}(t)$ may be not continuous, as long as $m^{2}(t)$ and the solutions exist $\forall t$. This will be the case here, as we will see below. Since $m^{2}(t<0)=0$, two independent equilibrium solutions are given for $t<0$ by

$$
h_{1}^{e q}(t, k)=\frac{i}{\sqrt{2 k}} e^{-i k t} ; \quad h_{2}^{e q}(t, k)=\frac{1}{\sqrt{2 k}} e^{i k t}
$$

which we have normalized so that $W^{e q}(t, k)=1$. It is not difficult to see that continuity and differentiability at $t=0$ imply that $W(t, k)=1$ also for $t>0$. On the other hand, since $m^{2}(t)$ is real, if $h_{1}(t)$ is a solution to Eq. (7), so it is $h_{2}(t)$ $=i h_{1}^{*}(t)$, where $W(t)=1$ if it matches the equilibrium solution at $t=0$.

We shall be dealing here with real time evolution for positive time coordinates and therefore, unless otherwise stated, we will be interested in $G^{11}$ only. In that case, we will suppress the " 11 " superscript for simplicity. Nonetheless, it should be borne in mind that in the loop integrals there are contributions from all the branches of the contour [28].

Thus, the solution for $G_{0}^{11}$ is given by [33]

$$
\begin{aligned}
i G_{0}\left(t, t^{\prime}, k\right)= & h_{1}(t, k) h_{1}^{*}\left(t^{\prime}, k\right) \theta\left(t-t^{\prime}\right) \\
& +h_{1}^{*}(t, k) h_{1}\left(t^{\prime}, k\right) \theta\left(t^{\prime}-t\right)+n_{B}(k) \\
& \times\left[h_{1}(t, k) h_{1}^{*}\left(t^{\prime}, k\right)+h_{1}^{*}(t, k) h_{1}\left(t^{\prime}, k\right)\right]
\end{aligned}
$$

where $t$ and $t^{\prime}$ are both positive and the boundary conditions at $t=0$ imply

$$
h_{1}\left(t=0^{+}, k\right)=\frac{i}{\sqrt{2 k}} ; \quad \dot{h}_{1}\left(t=0^{+}, k\right)=\sqrt{\frac{k}{2}}
$$

where the dot means $d / d t$. The dependence with the initial temperature appears through the Bose-Einstein distribution function

$$
n_{B}(k)=\frac{1}{e^{\beta_{i} k}-1} .
$$

In different parts of this work we will need the two-point function evaluated at the same space-time points:

$$
\begin{aligned}
G_{0}(t) & \equiv G_{0}(x, x) \\
& =\int \frac{d^{d-1} k}{(2 \pi)^{d-1}} G_{0}(t, t, k) \\
& =\frac{2}{\Gamma\left(\frac{d-1}{2}\right)(4 \pi)^{(d-1) / 2}} \int_{0}^{\infty} d k k^{d-2} G_{0}(t, t, k)
\end{aligned}
$$

where the equal-time correlator in momentum space reads, from Eq. (10):

$$
i G_{0}(t, t, k)=\left[1+2 n_{B}(k)\right]\left|h_{1}(t, k)\right|^{2} .
$$


Because of the loss of time translation invariance, $G_{0}(t)$ is a time-dependent quantity. Besides, it may be UV divergent and, therefore, we will use dimensional regularization (DR) with $d$ the space-time dimension, which is a suitable regularization scheme as far as chiral Lagrangians are concerned [16-18].

\section{Renormalization and curved space-time}

Once we have defined our nonequilibrium power counting, we can apply ChPT to calculate the time evolution of the observables. In doing so, we must pay special attention to renormalization. The fact that there is a time-dependent mass term indicates that there can be new time-dependent infinities in the chiral loops. For instance, in standard ChPT with a nonzero pion mass, the tadpoles renormalizing the pion propagator to lowest order yield the usual infinities proportional to $m_{\pi}^{2}$ in dimensional reduction (DR). These infinities are absorbed by two counterterms proportional to $m_{\pi}^{2}$ in the fourth order Lagrangian [16]. We expect similar divergent contributions here proportional to $\mathrm{m}^{2}(t)$. However, we are working in the chiral limit and therefore we are not allowed to introduce the above mentioned counterterms. Otherwise we would break explicitly the chiral symmetry. Hence, we should be able to construct the most general fourth order action, which in particular has to include new terms (and hence new low-energy constants) to cancel those extra divergences, preserving exactly the chiral symmetry.

There is a natural way to find this $\mathcal{O}\left(p^{4}\right)$ Lagrangian, using a very fruitful analogy: the action (1) can be written as a NLSM on a curved space-time background corresponding to a spatially flat Robertson-Walker (RW) metric, with scale factor $a(t)=f(t) / f$. For that purpose it is more convenient to work in terms of rescaled fields $\tilde{\pi}^{a}(\vec{x}, t)=\pi^{a}(\vec{x}, t) / a(t)$. Hence, $U=\left[\left(f^{2}-\tilde{\pi}^{2}\right)^{1 / 2} I+i \tau_{a} \tilde{\pi}^{a}\right] / f$ and we can write the action (1) as

$$
S_{2}[U]=\int_{C} d^{4} x \frac{f^{2}}{4} \sqrt{-g} g^{\mu \nu} \operatorname{tr} \partial_{\mu} U^{\dagger}(\vec{x}, t) \partial_{\nu} U(\vec{x}, t)
$$

where the metric $g^{\mu \nu}$ is nothing but the spatially flat RW metric with line element $d s^{2}=a^{2}(t)\left[d t^{2}-d \vec{x}^{2}\right]$ in conformal time $t[30]$ and $g \equiv \operatorname{det} g=-a^{8}(t)$.

With our chiral power counting, it is straightforward to assign the chiral order of the covariant tensors constructed from the metric. For instance, the Ricci tensor $R_{\mu \nu}$ is $\mathcal{O}\left(p^{2}\right)$ and so is the Ricci scalar $R=g^{\mu \nu} R_{\mu \nu}$, and so on. Explicit expressions for these tensors and other useful results for this metric are collected in Appendix B. An important point in this formulation is that we are considering the so called minimal coupling of the matter fields with the metric. That is, we are discarding possible couplings between the pion fields and $R(x)$ to $\mathcal{O}\left(p^{2}\right)$, such as $\xi R(x) \operatorname{tr}\left(U+U^{\dagger}\right)$. The reason is that we want to preserve chiral invariance, which would be broken by those terms [34]. Thus, in this language, $m^{2}(t)$ in Eq. (3) represents the minimal coupling with the RW metric preserving chiral invariance.
Therefore, we have a systematic way to construct the nonequilibrium Lagrangian to any order. We just have to include all possible terms consistent with the chiral symmetry, contracting indices covariantly with the metric $g_{\mu \nu}(x)$. In particular, to $\mathcal{O}\left(p^{4}\right)$ it reads [34]

$$
\begin{aligned}
S_{4}[U, g, R]= & \int_{C} d^{4} x \sqrt{-g}\left[\mathcal{L}_{4}[U, g]\right. \\
& \left.-\left(L_{11} R g^{\mu \nu}+L_{12} R^{\mu \nu}\right) \operatorname{tr} \partial_{\mu} U^{\dagger} \partial_{\nu} U\right]
\end{aligned}
$$

where $\mathcal{L}_{4}[U, g]$ stands for the standard (equilibrium) Lagrangian [16] with indices raised and lowered with the $g^{\mu \nu}$ metric and the rest are new $\mathcal{O}\left(p^{4}\right)$ invariant couplings with $R(x)$ and $R_{\mu \nu}(x)$ in the chiral limit. These are the new terms we need, where $L_{11}$ and $L_{12}$ are the new coupling constants. We are following the notation of [34], where this Lagrangian was first considered to study the energy-momentum tensor of QCD at low energies. ${ }^{1}$ The same Lagrangian has been used in the context of pion hard exclusive production [36]. In [34] it has been shown that in order to cancel the one-loop infinities, $L_{11}$ has to be renormalized as

$$
L_{11}=L_{11}^{r}(\mu)+\frac{1}{6} \frac{\mu^{d-4}}{16 \pi^{2}}\left[\frac{1}{d-4}-\frac{1}{2}(\log 4 \pi-\gamma+1)\right]
$$

with $d$ the space-time dimension, $\gamma$ the Euler constant and $\mu$ the renormalization scale. $L_{11}^{R}(\mu)$ is finite and depends on $\mu$ so that the combination in the right-hand side (RHS) of Eq. (17) remains scale-independent. On the other hand, $L_{12}$ is finite. Their numerical values can be obtained from the experimental information on the QCD energy-momentum form factors. They yield $L_{12} \simeq-2.7 \times 10^{-3}$ and $L_{11}^{r}(\mu=1 \mathrm{GeV})$ $\simeq 1.4 \times 10^{-3}$. We will use these same values here since their possible nonequilibrium corrections are of higher order in our analysis.

Thus in our case we have to replace in Eq. (16) the RW metric. After expanding in the $\pi^{a}$ fields and partial integration we have

$$
\begin{aligned}
S_{4}[\pi, g]= & -\frac{1}{2} \int_{C} d^{4} x \pi^{a}\left[f_{1}(t) \partial_{t}^{2}-f_{2}(t) \nabla^{2}+m_{1}^{2}(t)\right] \pi^{a} \\
& +\mathcal{O}\left(\pi^{4}\right)
\end{aligned}
$$

with

$$
f_{1}(t)=-12\left[\left(2 L_{11}+L_{12}\right) \frac{\ddot{f}(t)}{f^{3}(t)}-L_{12} \frac{[\dot{f}(t)]^{2}}{f^{4}(t)}\right]
$$

\footnotetext{
${ }^{1}$ The terms with $L_{11}$ and $L_{12}$ in Eq. (16) differ in a global sign from those in [34]. The reason is that we are following here the convention in [35] for the Riemann tensor, namely $R_{\beta \gamma \delta}^{\alpha}={ }_{{ }_{\delta}} \Gamma_{\beta \gamma}^{\alpha}$ $-\cdots$, where $\Gamma_{\beta \gamma}^{\alpha}$ are the Christoffel symbols (see Appendix B) whereas in [34] the convention for $R_{\beta \gamma \delta}^{\alpha}$ is reversed in sign. For the same reason, every term proportional to $L_{11}$ or $L_{12}$ here has its sign changed with respect to those in [28].
} 


$$
\begin{aligned}
& f_{2}(t)=-4\left[\left(6 L_{11}+L_{12}\right) \frac{\ddot{f}(t)}{f^{3}(t)}+L_{12} \frac{[\dot{f}(t)]^{2}}{f^{4}(t)}\right] \\
& m_{1}^{2}(t)=-\left[\frac{f_{1}(t) \ddot{f}(t)+\dot{f}_{1}(t) \dot{f}(t)}{f(t)}+\frac{1}{2} \ddot{f}_{1}(t)\right] .
\end{aligned}
$$

The above Lagrangian should take care of the nonequilibrium infinities we might find in the pion two-point function. As far as this work is concerned, these are the only infinities we will have to renormalize.

In the following sections we will concentrate on a particular case for $f(t)$ (or the scale factor if we use the curved space time terminology) which is of physical relevance as the simplest approximation producing a large number of correlated pions. In addition, this example will allow us to test explicitly the cancellation of the (new) nonequilibrium infinities appearing in the observables considered.

\section{PARAMETRIC RESONANCE AND PION PRODUCTION}

\section{A. The parametric resonance approach}

It is clear that our approach will be useful in a stage of the plasma evolution when the departure from equilibrium is of the same order as the meson energies. Hence, as far as pion production is concerned, we are in the parametric resonance regime. Let us briefly review some of the ideas behind parametric resonance in the $O(4)$ model $[14,27,9]$. In the last stage of the field evolution, the $\sigma$ field is oscillating near the true vacuum and those oscillations have relatively small amplitude [23]. Following a semiclassical approach, one can split the $\sigma$ field as $\sigma(\vec{x}, t)=\sigma_{0}(t)+\delta \sigma(\vec{x}, t)$ where $\sigma_{0}(t)$ is a time-dependent homogeneous classical background, solution of the equations of motion to leading order in the amplitude, whereas $\delta \sigma$ includes next to leading order corrections and quantum fluctuations. One can proceed perturbatively around the classical solution $\sigma_{0}(t)$. In a first approximation, quantum fluctuations of both the $\sigma$ and the pions can be neglected. Thus, if the $\sigma$ field is oscillating around the potential minimum $\sigma=f$ and the amplitude of the oscillations $q$ is small, one can solve the equations of motion perturbatively in $q$. To leading order the equations of motion for the $\sigma$ and the pions decouple from each other and one simply gets $\sigma_{0}(t) / f=1-(q / 2)\left[\sin \left(m_{\sigma} t+\varphi\right)-\sin \varphi\right]$ where $m_{\sigma}$ is the $\sigma$ mass, $\varphi$ is an arbitrary phase and we have chosen the initial conditions so that the field is at the bottom of the potential for $t=0$, consistently with our choice of the initial equilibrium state. Neglecting the pion correlations is equivalent to state that $\left\langle\pi^{2}\right\rangle \ll f^{2}$. If the first order solution is inserted into the equations of motion to next to leading order, the pions satisfy a Mathieu equation. The importance of this equation is that it has solutions exponentially growing in time for certain bands in momentum space. This is the essence of the parametric resonance mechanism, which even in this simple classical picture is consistent with hadronization [14]. The parametric resonance idea is directly imported from reheating and preheating in inflationary cosmology [24], where the small $q$ approach is called the narrow resonance limit for reasons to become clear below.

The approach described in the previous paragraph is the crudest one can follow in this context, although it reproduces the main features of parametric resonance. One can refine it in several ways. First, neglecting the pion correlations but keeping the NLO terms in the $\sigma$ amplitude leads to a Lamé equation instead of the Mathieu equation [27,26]. As it is emphasized in [27], the difference is not only quantitative, but the resonance structure is also different. As for the importance of pion correlations, one must bear in mind that this is a matter of time scales. Such correlations grow exponentially in time until eventually they reach the same order of magnitude as the $\sigma$ term. As it is customary, we will refer to that time scale as the back-reaction time, so that, typically $\left\langle\pi^{2}\right\rangle\left(t_{B R}\right) \sim f^{2}$. The name is again inherited from Cosmology where the back-reaction describes the modifications of the metric or the inflaton field due to quantum fluctuations of the matter fields $[24,30]$. It is clear that for $t \geqslant t_{B R}$, pion correlations must be included self-consistently in the dynamics of the $\sigma$ field. In $O(N)$ models this usually requires numerical simulations. For instance, in the large $N$ limit it has been shown that, when the back-reaction is properly accounted for, pion correlations are responsible for the damping of the $\sigma$ field from $t_{B R}$ onwards and, more importantly, this dissipation stops the exponential growth in the pion number $[27,37]$. A different story though is that one can interpret that process as thermalization, or in other words, that the final particle spectrum is thermal. In fact, that is not the case when pion amplification occurs [23]. Thus, in practice, all the interesting physics associated with pion production in parametric resonance takes place before the back-reaction time. Finally, a word must be said about the quantum corrections of the $\sigma$ field, which can be treated semiclassically [9]. In the narrow resonance approach, the evolution of the pion fluctuations is influenced only by terms linear in $\sigma_{0}(t)$ and the dynamics of the $\sigma$ fluctuations is not important for pion production, since the width of the resonance band for the $\sigma$ is negligible with respect to that of the pions. Thus, the essence of the exponential growing of pion fluctuations is not changed qualitatively by including quantum corrections in $\delta \sigma$.

At this point, let us establish the connection with our present ChPT approach. Our philosophy will be to work out consistently the simplest choice for $f(t)$ yielding parametric resonance. For that purpose, it is useful to compare with the $O(4)$ model. In the oscillatory regime (with small oscillations) one starts by keeping only the leading order $\sigma$ $\sim \sigma_{0}(t)$. In that limit and for small oscillations the NLSM is nothing but the $O(4)$ model subject to $\pi^{2}+\sigma^{2}=\sigma_{0}^{2}(t)$. In other words, we should take simply

$$
f(t)=f\left[1-\frac{q}{2} \sin M t\right] \quad(t>0)
$$

where we have chosen $\varphi=0$ so that

$$
m^{2}(t)=-\left(q M^{2} / 2\right) \sin M t+\mathcal{O}\left(q^{2}\right)
$$


Note that with this choice, not only $f\left(0^{+}\right)=f$ but also $m^{2}\left(0^{+}\right)=0$, i.e, both $f(t)$ and $m^{2}(t)$ match their equilibrium values at $t=0$. This will play an important role in the analysis in Sec. III B.

Thus, we would use the NLSM model (1) with $f(t)$ in Eq. (20) if we were interested in describing pions out of equilibrium classically, for times where the back reaction is not important and the plasma is in the broken phase. Then, we can calculate pion observables using ChPT, where the pion fluctuations can be treated quantum mechanically in a consistent fashion. As a matter of fact, there is no need to invoke the $O(4)$ model in the first place, since the NLSM is the lowest order action compatible with all the symmetries, driven out of equilibrium through the time dependence in $f(t)$. For that reason, we have replaced the mass of the sigma by $M$, an arbitrary mass parameter. However, we will see below that in order to obtain numerical results compatible with the expected values for the plasma lifetime and pion production, $M$ will be typically around the phenomenological values of $m_{\sigma}$ used in the $O(4)$ model. The advantage of our present approach is that we do not have to worry about the uncertainties related to the $\sigma$ particle. $^{2}$

Let us be more precise now about the smallness of $q$ in our approach. According to our previous discussion about the nonequilibrium chiral power counting, we should demand at least that $q M^{2}=\mathcal{O}\left(p^{2}\right)$ and so on. In this way, all the $\mathcal{O}\left(p^{4}\right)$ corrections will remain under control, as we will see below. Note that, according to the discussion in the previous paragraph, if we think of $M \sim m_{\sigma}$, our chiral power counting is equivalent to $q=\mathcal{O}\left(p^{2} / \Lambda_{\chi}^{2}\right)$. It is important to remark that in this work we shall restrict to one loop in ChPT, where we will see that the above power counting is consistent for the typical values of $M$ considered here. Going beyond that could imply additional restrictions on the value of $M$ and the power counting. Before carrying on, we would like to summarize the assumptions and the limitations of the present approach:

(i) We are neglecting the possible back-reaction corrections to $f(t)$ in Eq. (20). This is valid for times below $t_{B R}$, when the pion correlations are of the same order as the leading order, that is, $\left\langle\pi^{2}\right\rangle\left(t_{B R}\right) \sim f^{2}$. However, we will see in Sec. IV that within our approach one can estimate $t_{B R}$ by calculating the loop corrections to $f_{\pi}(t)$. Fortunately, as we said before, nearly all the relevant nonequilibrium pion production physics happens before that time. This limitation comes from the fact that we are treating $f(t)$ as external, similarly to quantum field theory in an external curved space-time, as we have seen in Sec. II C. A self-consistent approach, analogous to treat also the metric quantummechanically in a perturbative low-energy fashion would be very interesting but is out of the scope of this work.

\footnotetext{
${ }^{2}$ The $\sigma$ mass is not very well determined. It ranges between $400-$ $1200 \mathrm{MeV}$ according to the latest Particle Data Group (PDG) data [38]. It is not even clear that one can describe it as a particle. For instance, it shows up in pion-pion scattering in ChPT as a rather broad resonance in the $I=J=0$ channel [39].
}

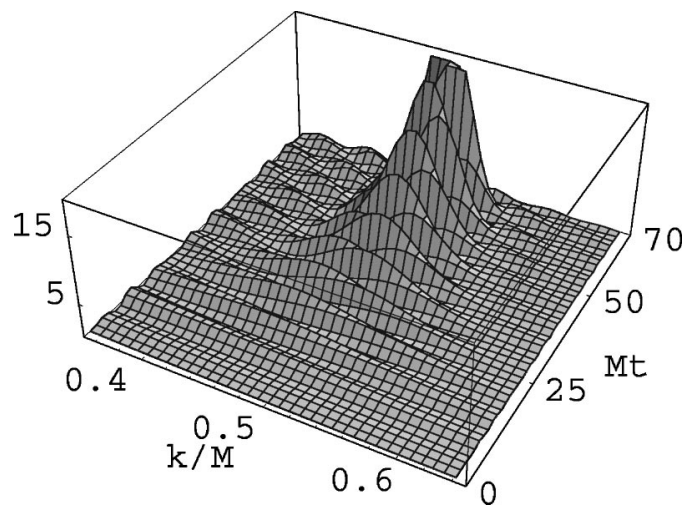

FIG. 2. Profile of $i M G_{0}(t, t, k)$ for $T_{i}=M / 100$ and $q=0.1$. The instability band for this case lies roughly between $0.47 M<k<0.52 M$.

(ii) We are assuming that the system is the late stage of the expansion, so that it makes sense to treat the amplitude of the oscillations $q$ as a small parameter (narrow resonance approximation). That means we will only retain the leading order in $q$ and thus ignore $\mathcal{O}\left(q^{2}\right)$ corrections. This is consistent with ChPT to one-loop if $q M^{2}=\mathcal{O}\left(p^{2}\right)$. We will see that this simplification amounts, among other things, to consider the Mathieu equation for the pion modes.

(iii) We shall restrict to one loop in ChPT, in the chiral limit and for the $S U(2)$ chiral symmetry. As commented before, the nature of the ChPT approach allows to extend our calculations including quark masses and three flavors.

\section{B. Dimensional regularization of the LO propagator}

According to the previous discussion, the differential equation (7) becomes to leading order in $q$, the Mathieu equation:

$$
\frac{d^{2} h_{1}(z, k)}{d z^{2}}+[a(k)-2 q \cos 2 z] h_{1}(z, k)=0
$$

where $z=M t / 2-\pi / 4, a(k)=4 k^{2} / M^{2}$ and, without loss of generality, we will take $q>0$.

The solutions of the Mathieu equation are known and tabulated. We have collected in Appendix A some useful results about this equation. For our purposes, the most relevant feature is that it admits unstable solutions exponentially growing in time for certain values of the parameter $a$. This is the simplest version of the parametric resonance mechanism. The instabilities arise in bands in $k$, centered at $k_{n} \simeq n M / 2$, of width $\Delta k_{n}=\mathcal{O}\left(q^{n}\right)$ (see Appendix A). Therefore, in the narrow resonance approximation we will just neglect the width of all the bands but the first one. A typical unstable solution for the equal-time correlation function $G_{0}(t, t, k)$ has been plotted in Fig. 2 around the first band for a particular choice of the parameters. The solutions typically oscillate with an exponentially growing amplitude inside the unstable region. These will be the field configurations responsible for explosive pion production.

Our next step will be to analyze the equal-time correlator $G_{0}(t)$ in parametric resonance, separating its UV divergent 
part in DR. Therefore, we need to know the behavior of $G_{0}(t, t, k)$ in Eq. (14) for large $k$. Since we are considering only one resonant band in momentum space then $G_{0}(t, t, k)$ is in the stable region for large enough $k$. For small $q$, the analytic solution in the stable zone is given in Eq. (A6). Let us define $k_{0}$ such that for $k>k_{0}(>M / 2)$ one can simply take $\tilde{q}=q /[2(a-1)]$ in Eq. (A7). As explained in Appendix A, it is not difficult to estimate numerically the value of $k_{0}$ for a given $q$. We have found that $k_{0}=M$ satisfies the above requirement within our approximation range for $q \leqslant 1$. Nevertheless, we have checked that our results do not depend on the choice of $k_{0}$ as long as $k_{0} \geqslant M$.

Thus, replacing the approximate solution (A6) in Eq. (A5), solving for the coefficients $A(k)$ and $B(k)$ and replacing the result in Eq. (14) we find, to leading order in $q$,

$$
\begin{aligned}
i G_{0}(t, t, k)= & \frac{1}{2 k}\left[1+2 n_{B}(k)\right] \\
& \times\left\{1+\frac{q M^{2}}{4} \frac{1}{k^{2}-M^{2} / 4}\left[\sin M t-\frac{M}{2 k} \sin 2 k t\right]\right\}
\end{aligned}
$$

for $k \geqslant k_{0}$. First, let us split the $k$ integral in Eq. (13) as

$$
\int_{0}^{\infty}=\int_{0}^{k_{0}}+\int_{k_{0}}^{\infty}
$$

The first piece is finite so that we can take $d=4$ and use either the asymptotic or numerical solutions for the Mathieu equation. In the second, which is UV divergent, we replace the solution (22). It is clear that the piece proportional to the Bose-Einstein function is UV finite, since $n_{B}(k)$ decreases exponentially for large $k$. In addition, the integral of any power of $k$ is identically zero in DR, so that $\int_{k_{0}}^{\infty} d k k^{d-3}=$ $-\int_{0}^{k_{0}} d k k^{d-3}$ and we can absorb that contribution into the first piece in Eq. (23). ${ }^{3}$ Therefore, let us write for $t>0$ :

$$
\begin{aligned}
i G_{0}(t)= & \frac{T_{i}^{2}}{12}-\frac{q M^{2}}{32 \pi^{2}} I(t)+i G_{0}^{d i v}(t) \\
& +\frac{1}{2 \pi^{2}}\left\{\int_{0}^{k_{0}} d k k^{2}\left[i G_{0}(t, t, k)-\frac{1}{2 k}\left[1+2 n_{B}(k)\right]\right]\right. \\
& \left.+\frac{q M^{2}}{4} \int_{k_{0}}^{\infty} d k k n_{B}(k) \frac{\sin M t-\frac{M}{2 k} \sin 2 k t}{k^{2}-M^{2} / 4}\right\}
\end{aligned}
$$

where we have separated explicitly the equilibrium contribution which is just

\footnotetext{
${ }^{3}$ The above result can be understood formally in DR by taking $\lim _{m \rightarrow 0} \int_{k_{0}}^{\infty} k^{d-1} /\left(k^{2}+m^{2}\right)=-k_{0}^{d-2} /(d-2)$.
}

$$
i G_{0}^{e q}=\frac{1}{2 \pi^{2}} \int_{0}^{\infty} d k k \frac{1}{e^{\beta_{i} k}-1}=\frac{T_{i}^{2}}{12}
$$

and the integral

$$
I(t)=M \int_{k_{0}}^{\infty} d k \frac{\sin 2 k t}{k^{2}-M^{2} / 4}
$$

is finite for $t \geqslant 0$. It is important to point out that if we had chosen a different phase $\varphi$ in $f(t)$, as for instance $f(t)$ $=f[1-(q / 2)(\cos M t-1)]$, we would have found a singular behavior near $t=0$. In fact, instead of $I(t)$ above, we would have an integral which is finite for $t>0$ and $d \rightarrow 4$ but logarithmic divergent in the $t \rightarrow 0^{+}$limit. In this sense, $t$ acts as a natural regulator. This would not have been a limitation to our approach, since we are meant to observe the system for times such that the $t=0$ effects are unimportant. In fact, we have checked numerically that the influence of those terms is irrelevant for $M t \gtrsim 1$. The behavior at $t=0$ is just a consequence of our non-analytic approach, where the nonequilibrium effects appear instantaneously and is a well-known problem in nonequilibrium field theory. In fact, in [40] it has been pointed out that it can be cured by a suitable choice of the initial state, which for a time-dependent mass term amounts to take $\mathrm{m}^{2}\left(0^{+}\right)$equal to the initial mass. This is exactly what we have done with our choice of phase in Eq. (20), since $m^{2}\left(0^{+}\right)=0$ in the chiral limit. Our results confirm the analysis in [40] from a completely different viewpoint, namely working in path integral within the ChPT framework in the DR scheme.

The divergent part in Eq. (24) is given by

$$
i G_{0}^{d i v}(t)=\frac{\sin M t}{\Gamma\left(\frac{d-1}{2}\right)(4 \pi)^{(d-1) / 2}} \frac{q M^{2}}{4} \int_{k_{0}}^{\infty} d k \frac{k^{d-3}}{k^{2}-M^{2} / 4}
$$

We will proceed now to regularize this expression. We have [41]

$$
\begin{aligned}
\int_{k_{0}}^{\infty} d k \frac{k^{d-3}}{k^{2}-M^{2} / 4} & =\frac{k_{0}^{d-4}}{4-d^{2}}{ }_{2} F_{1}\left[1,2-\frac{d}{2} ; 3-\frac{d}{2} ; \frac{M^{2}}{4 k_{0}^{2}}\right] \\
& =\frac{\left(k_{0}^{2}-M^{2} / 4\right)^{(d-4) / 2}}{4-d}+\mathcal{O}(d-4),
\end{aligned}
$$

which replaced in Eq. (27) yields

$$
\begin{aligned}
i G_{0}^{d i v}(t)= & -\frac{q M^{2}}{32 \pi^{2}}\left\{2\left(k_{0}^{2}-M^{2} / 4\right)^{(d-4) / 2} \sin M t\right. \\
& \left.\times\left[\frac{1}{d-4}-\frac{1}{2}(\log \pi-\gamma+2)\right]+\mathcal{O}(d-4)\right\} .
\end{aligned}
$$


As we will see in detail in Sec. IV, we will be able to renormalize this divergence in the low-energy constant $L_{11}$ so that the answer for the observables is finite and scale independent. This means that the regularization of the above UV divergence is consistent.

\section{THE PION DECAY FUNCTIONS $f_{\pi}(t)$ TO ONE LOOP}

The pion decay constant acquires one-loop corrections in ChPT. Those corrections are finite and scale-independent once the contribution from the $O\left(p^{4}\right)$ Lagrangian is taken into account [16]. The same will happen in our nonequilibrium model, where the pion decay constant becomes a timedependent function $f_{\pi}(t)$, which to tree level is just $f(t)$. For us, the importance of the calculation of $f_{\pi}(t)$ is twofold: first, it will provide an explicit check of consistency of our renormalization scheme. Second, it will help us to understand the time scales. In particular, the size of the loop correction will define the back-reaction time, when it equals the tree level contribution. For times well below that scale, our approach remains perfectly valid and yields predictions for observables such as $f_{\pi}(t)$ and the pion number. As a matter of fact, the same philosophy is followed at finite temperature in equilibrium, where in the chiral limit [19]:

$$
\left[f_{\pi}^{2}(T)\right]=f^{2}\left(1-\frac{T^{2}}{6 f_{\pi}^{2}}\right)
$$

with $f_{\pi} \simeq 93 \mathrm{MeV}$. Clearly, this result is valid only for temperatures below $T^{*}=\sqrt{6} f_{\pi} \simeq 228 \mathrm{MeV}$. In fact, even though $f_{\pi}(T)$ is not a good order parameter for the chiral phase transition [21], it should decrease as $T$ approaches the critical temperature $T_{c}$. Therefore, the one-loop result (30) already reproduces the correct qualitative behavior and indeed it provides a reasonable estimate $T^{*}$ for the critical temperature. The $T^{2}$ term in Eq. (30) is nothing but the thermal pion correlator $\left\langle\pi^{2}(0)\right\rangle$ in the chiral limit in Eq. (25) and at temperatures near $T^{*}$ pion correlations are of the same size as $f^{2}$ $\left[\sim\left\langle\sigma^{2}\right\rangle\right.$ in the $O(4)$ model $]$ so that higher order corrections become equally important.

We should bear in mind that the definition of $f_{\pi}$ is subtle even in thermal equilibrium. In fact, it is more convenient to define it as the residue of the axial-axial thermal spectral function $\left\langle T_{C}\left[A_{\mu}^{a}(x), A_{\mu}^{b}(y)\right]\right\rangle[21,42]$, where $A_{\mu}^{a}(x)$ is the axial current $(a=1,2,3)$, instead of using the PCAC theorem [17]. In this way, one avoids dealing with the reduction formula and asymptotic states at finite temperature. In addition, it is important to bear in mind that, due to the loss of Lorenz covariance in the thermal bath, one can define two different pion decay constants $f_{\pi}^{s}$ and $f_{\pi}^{t}$ corresponding, respectively, to the space and time components of the axial current [43]. In fact, the chiral symmetry imposes relations between them and the in-medium pion dispersion law. If the chiral symmetry is exact (as it is in our case) pions remain massless but their velocity $v_{\pi}$ can be less than the speed of light and their thermal width can be different from zero. The relation with $f_{\pi}^{s, t}$ is given by $v_{\pi} \simeq \operatorname{Re} f_{\pi}^{s} / \operatorname{Re} f_{\pi}^{t}$, while the thermal width is proportional to the imaginary parts of $f_{\pi}^{s}$ and $f_{\pi}^{t}$ [43]. Nev- a)

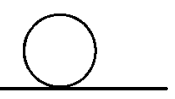

b)

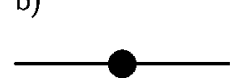

c)

FIG. 3. The different diagrams contributing to the pion decay functions to NLO in ChPT.

ertheless, to one loop in ChPT one has $\operatorname{Im} f_{\pi}^{s}=\operatorname{Im} f_{\pi}^{t}=0$ and $f_{\pi}^{s}=f_{\pi}^{t}=f_{\pi}(T)$ in Eq. (30). Beyond one loop, the $\mathcal{O}\left(T^{4}\right)$ velocity corrections are nonzero and have been calculated in ChPT $[44,45]$. The $\mathcal{O}\left(T^{4}\right)$ corrections also appear in the $O$ (4) model to one loop [43]. As for the imaginary part, it is also different from zero beyond one loop. The corresponding pion damping rate has also been calculated in [45], in agreement with previous analysis in the literature $[46,47]$. As we are going to see, in the present model we will get a small but nonzero difference $f_{\pi}^{s}-f_{\pi}^{t}$ to one-loop, unlike equilibrium, which could be interpreted as a small nonequilibrium deviation for the pion velocity. The pion velocity plays also an important role in the hydrodynamics of the chiral phase transition [29].

\section{A. Nonequilibrium pion decay functions}

All the above considerations for $f_{\pi}$ can be extended to nonequilibrium. We refer to our earlier work [28] for further details. The axial current from Eq. (1) reads

$$
A_{\mu}^{a}(\vec{x}, t)=i \frac{f^{2}(t)}{4} \operatorname{tr}\left[\tau^{a}\left(U^{\dagger} \partial_{\mu} U-U \partial_{\mu} U^{\dagger}\right)\right] .
$$

As in equilibrium, there are two independent $f_{\pi}^{s}(t)$ and $f_{\pi}^{t}(t)$. Their definitions are given in [28] consistently with the Ward identities of chiral symmetry. To leading order in ChPT [tree level with the action (1)] one has to consider only $\mathcal{O}(\pi)$ contributions when expanding the $U$ fields in the axial current (31) which yields, as it should, $f_{\pi}^{s}(t)=f_{\pi}^{t}(t)=f(t)$ at tree level.

To next-to-leading order (NLO) there are three different contributions to $f_{\pi}$ : the first one comes from the NLO corrections to the propagator. Such corrections are of two types: one loop diagrams from the $S_{2}$ action and tree level ones from $S_{4}$ [diagrams (a) and (b) in Fig. 3 respectively]. It is important to bear in mind that in the calculation of diagram (a) one has to integrate over all the branches of the contour $C$ in Fig. 1 and the result is independent of $t_{i}$. Second, there is another one-loop diagram involving the product of three pion fields at the same space-time point [diagram (c)] in Fig. 3 from the next order in the expansion of the axial current. Finally, at the same order we have to take into account that the axial current in Eq. (31) is itself modified by the fourth order Lagrangian in Eq. (16) as $\left[1+f_{1}(t)\right] A_{0}$ and $[1$ $\left.+f_{2}(t)\right] A_{j}$ with $f_{1}(t)$ and $f_{2}(t)$ in Eq. (19).

Once the different contributions have been taken into account, the final result to $\mathcal{O}\left(p^{4}\right)$ reads [28]

$$
\begin{aligned}
& {\left[f_{\pi}^{s}(t)\right]^{2}=f^{2}(t)\left[1+2 f_{2}(t)-f_{1}(t)\right]-2 i G_{0}(t)} \\
& {\left[f_{\pi}^{t}(t)\right]^{2}=f^{2}(t)\left[1+f_{2}(t)\right]-2 i G_{0}(t)}
\end{aligned}
$$


for $t>0$, with $G_{0}(t)$ the equal-time correlation function defined in Eq. (13). The results (32),(33) reproduce the equilibrium result in Eq. (30) when the time derivatives of $f(t)$ are switched off and $G_{0}^{e q}$ is replaced by Eq. (25).

As in standard ChPT, the loop corrections to $f_{\pi}$ come directly from the equal time pion correlator, which is timedependent now. In addition, there are $\mathcal{O}\left(p^{4}\right)$ tree level corrections given by the terms proportional to $f_{1}(t)$ and $f_{2}(t)$. In the chiral limit in equilibrium there are no $\mathcal{O}\left(p^{4}\right)$ tree level corrections because $G_{0}^{e q}$ is finite. However, at nonequilibrium $G_{0}(t)$ is UV divergent as we have seen in Sec. III and the counterterms proportional to $f_{1,2}(t)$ are precisely those needed to arrive to a finite answer. The above result for $f_{\pi}(t)$ should be such that the total answer is finite and scale independent because $f_{\pi}$ is an observable. Thus, we should be able to absorb the UV infinities and the scale dependence (in DR) in the new low-energy constant $L_{11}$, as discussed in Sec. II C. In addition, from Eqs. (32),(33) and (19) we see that the difference $\left[f_{\pi}^{s}(t)\right]^{2}-\left[f_{\pi}^{t}(t)\right]^{2}$ remains finite (it depends only on $L_{12}$ ) so that the same renormalization is valid for $f_{\pi}^{s}(t)$ and $f_{\pi}^{t}(t)$, which is another consistency check. In fact, the above result yields $f_{\pi}^{s}(t) \neq f_{\pi}^{t}(t)$ to one loop, unlike the equilibrium case. Therefore, the plasma expansion induces modifications in the pion velocity larger than in equilibrium. However, note that we are following the equilibrium arguments given in [43] in order to relate a nonzero value for $f_{\pi}^{s}-f_{\pi}^{t}$ with the in-medium pion velocity. Thus, our conclusions in this respect must be taken with care. In Sec. IV C we will come back to this point and give some numerical estimates.

\section{B. $f_{\pi}^{s}(t)$ and $f_{\pi}^{t}(t)$ in parametric resonance}

Let us concentrate now in the parametric resonance approach, with $f(t)$ given in Eq. (20) and where we keep only the leading order in the amplitude of the oscillations $q$. The loop contribution is given by $G_{0}(t)$, which we have analyzed in detail in Sec. III. It will grow exponentially in time due to explosive pion production, once the infinities have been suitably subtracted, whereas the tree level corrections $f_{1,2}(t)$ remain bounded in time.

The first step is to show how the infinities cancel in the final answer for $f_{\pi}(t)$. For that purpose, we replace in Eqs. (32),(33) the functions $f_{1,2}(t)$ in Eq. (19) to leading order in $q$. On the other hand, in Sec. III we have regularized the equal-time two-point function in DR. Its divergent divergent part for $d \rightarrow 4$ is given in Eq. (29). According to our previous discussion, we should be able to absorb the divergent part in $L_{11}$. In fact, collecting the piece proportional to $L_{11}$ in both $f_{\pi}^{s, t}(t)$ (remember that they only differ in terms proportional to $L_{12}$ ) plus the divergent contribution in Eq. (29) and using Eq. (17) yields

$$
\begin{aligned}
{\left[f_{\pi}(t)^{d i v}\right]^{2}=} & -q M^{2} \sin M t \\
& \times\left[12 L_{11}^{r}(\mu)+\frac{1}{16 \pi^{2}}\left(1+\log \frac{\mu^{2}}{4 k_{0}^{2}-M^{2}}\right)\right]
\end{aligned}
$$

where we have taken the $d \rightarrow 4$ limit. The above contribution is finite and scale independent [the explicit dependence with $\mu$ is compensated by that in $L_{11}^{r}(\mu)$ as explained before] which is a very important consistency check of our approach. Notice that it is crucial that the divergent contribution in Eq. (29) has exactly the same time dependence as $\ddot{f}(t) / f(t)$ in Eq. (19).

Therefore, collecting the various pieces above, we can write the final result for $f_{\pi}^{s}(t)$ and $f_{\pi}^{t}(t)$ in parametric resonance (to leading order in $q$ and to one loop in ChPT) as

$$
\begin{aligned}
& \frac{f_{\pi}^{s}(t)}{f_{\pi}\left(T_{i}\right)}= 1-\frac{q}{2} \sin M t+\frac{q M^{2}}{f_{\pi}^{2}}\left\{\frac{I(t)}{32 \pi^{2}}+\sin M t\right. \\
& \times\left[L_{12}-6 L_{11}^{r}(\mu)\right. \\
&\left.\left.-\frac{1}{32 \pi^{2}}\left(1+\log \frac{\mu^{2}}{4 k_{0}^{2}-M^{2}}\right)\right]-\Delta_{B}(t)\right\}-\frac{\Delta_{u n s t}(t)}{f_{\pi}^{2}} \\
& f_{\pi}^{t}(t)=f_{\pi}^{s}(t)\left(L_{12} \rightarrow-L_{12}\right)
\end{aligned}
$$

for $t>0$, with $f_{\pi}(T)$ in Eq. (30), $I(t)$ in Eq. (26) and

$$
\begin{gathered}
\Delta_{B}(t)=\frac{1}{8 \pi^{2}} \int_{k_{0}}^{\infty} d k k n_{B}(k) \frac{\sin M t-\frac{M}{2 k} \sin 2 k t}{k^{2}-M^{2} / 4} \\
\Delta_{\text {unst }}(t)=\frac{1}{2 \pi^{2}} \int_{0}^{k_{0}} d k k^{2}\left[i G_{0}(t, t, k)-\frac{1}{2 k}\left[1+2 n_{B}(k)\right]\right] .
\end{gathered}
$$

Note that the only unstable (exponentially growing) contribution in Eq. (35) is given by $\Delta_{u n s t}(t)$ in Eq. (38). The rest is bounded in time. Therefore, according to our previous discussion, we can estimate the back-reaction time as $\Delta_{\text {unst }}\left(t_{B R}\right) \simeq f_{\pi}^{2}$. Thus, we have an approximate idea of the time scale during which our one-loop approach can be trusted. From that time onwards, the back-reaction corrections to $f(t)$ coming from the coupling to the pion fields cannot be ignored.

\section{Numerical results}

Since the result is independent of the scale, we will fix $\mu=1 \mathrm{GeV}$ and use the numerical values of $L_{11}$ and $L_{12}$ determined phenomenologically in [34] and given in Sec. II C. We have also taken $k_{0}=M$ (see our previous comments).

There are still three parameters we have to fix corresponding to the initial conditions: the initial amplitude of the oscillations $q$, the initial frequency $M$ and the initial temperature $T_{i}$. According to our previous comments, $M$ should be around the value of the $\sigma$ mass in the $O(4)$ model, although in the present approach it is not necessary to assume the 

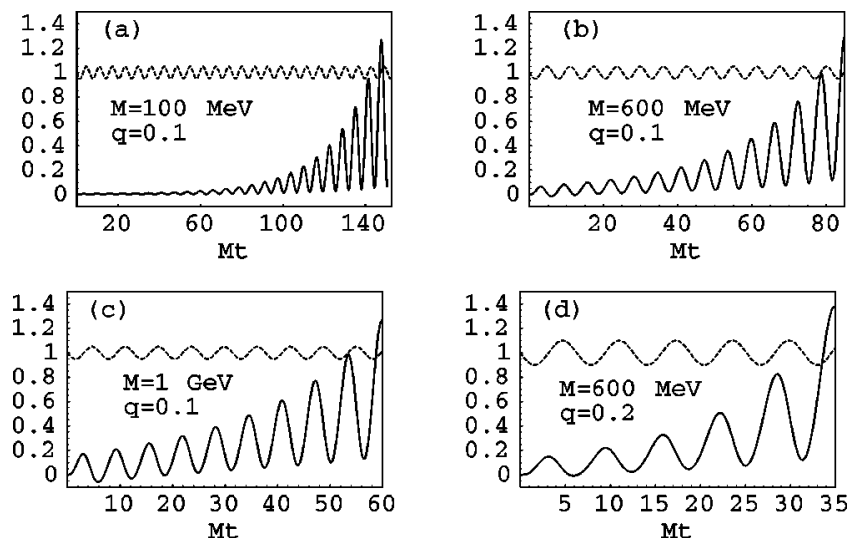

FIG. 4. The solid line is $\Delta_{\text {unst }}(t) / f_{\pi}^{2}$. The dashed line is the tree level contribution $1-q / 2 \sin M t . T_{i}=50 \mathrm{MeV}$ for all cases. The values of the back-reaction time are approximately given by (a) $t_{B R} \simeq 300 \mathrm{fm} / c$, (b) $t_{B R} \simeq 28 \mathrm{fm} / c$, (c) $t_{B R} \simeq 11 \mathrm{fm} / c$ and (d) $t_{B R}$ $\simeq 10 \mathrm{fm} / c$.

existence of a $\sigma$ particle. We have considered three different cases: $M=0.1,0.6$ and $1 \mathrm{GeV}$. The second value is the one more often used in the literature and also the closest one to the recent determinations of $m_{\sigma}$ [39]. As for the initial temperature, we have fixed for definiteness $T_{i}=50 \mathrm{MeV}$. The standard approach is to assume an initially supercooled (zero temperature) state when the $\sigma$ field starts its rolling down from the top of the potential. In this process the initial potential energy is converted into thermal energy of the pion gas (reheating) [14]. Hence, it is reasonable to assume in our case a nonzero but small initial temperature. Even though our model does not have a direct interpretation in terms of an effective potential, it corresponds in the language of the $O$ (4) model to an initial condition where the $\sigma$ has already reached the bottom of the potential and it is oscillating around it. Nonetheless we must point out that the results depend very weakly on $T_{i}$. The reason is that in the right hand side of Eq. (35) the dependence with $T_{i}$ enters through the integrals (37),(38) which are dominated by contributions near $k \simeq M$ and are therefore strongly damped by the BoseEinstein distribution $n_{B}$ for temperatures $T_{i} \ll M$. We have checked that taking different values for $T_{i}$ in the range 10$100 \mathrm{MeV}$ the curves showed below remain almost unchanged. Finally, we have considered $q=0.1$ and $q=0.2$ to illustrate the dependence of our results with the initial amplitude. The initial values we are considering here are similar to those used in the literature $[9,14,26]$.

The results for $\Delta_{\text {unst }}(t) / f_{\pi}^{2}$ are plotted in Fig. 4 . The values of the estimated $t_{B R}$ are also given in that figure. One clearly observes that increasing either $q$ or $M$ makes the unstable modes grow faster and overcome earlier the tree level value. In fact, the upper envelope of the long-time oscillations is proportional to $\left[q M^{2} /\left(4 \pi f_{\pi}\right)^{2}\right] \exp (q M t / 2)$ since, from Eqs. (A8) and (14), the dominant exponential contribution to the two point function at long times is $\exp (2 \mu z)$, the maximum of the Floquet exponent $\mu$ in Eq. (A9) being $\mu$ $\simeq q / 2$ at the center of the unstable band, which has width $q$.

The time it takes for the pion correlations to overcome the tree level has been estimated in the $O(4)$ model in this re-
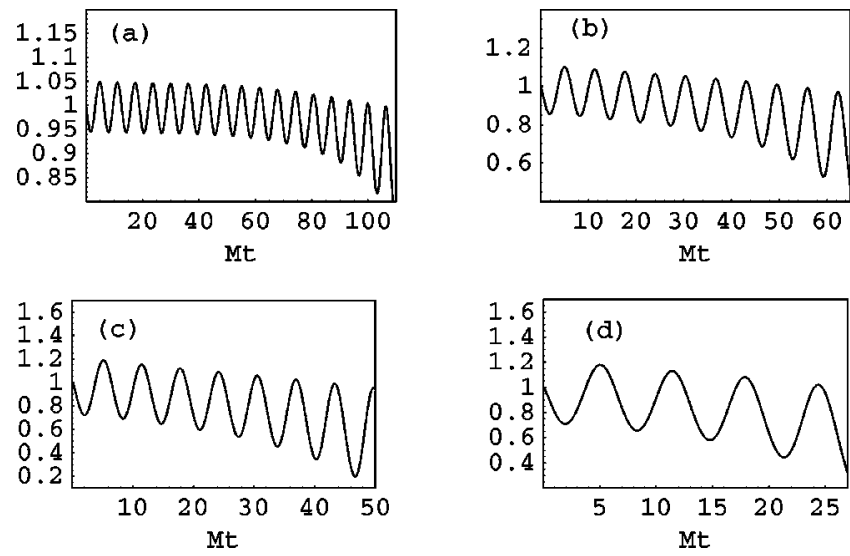

FIG. 5. $f_{\pi}^{s}(t) / f_{\pi}\left(T_{i}\right)$ for the same cases as in Fig. 4.

gime and it lies between $5-10 \mathrm{fm} / c$ [9,26]. As explained before, this is the same time scale as that when dissipation makes pion production stop. In the four cases we have considered, it is clear that our approach remains valid for the time relevant to pion production. For definiteness, we will restrict from now on to the choice of parameters (d) in Fig. 4 giving $t_{B R} \simeq 10 \mathrm{fm} / c$, which is of the order of the plasma lifetime. In turn, note that for cases (c) and (d) we have $q M^{2} / \Lambda_{\chi}^{2} \simeq 0.07$ and $q M^{2} / \Lambda_{\chi}^{2} \simeq 0.05$ respectively, so that our ChPT approach to $\mathcal{O}(q)$ is perfectly valid.

In Fig. 5 we have depicted the total result for $f_{\pi}^{s}(t) / f_{\pi}\left(T_{i}\right)$ in Eq. (35) for the same cases as in Fig. 4. In this curve, the upper limit in time corresponds roughly to the onset of the back reaction. Its effect is to make the amplitude of the oscillations grow, whereas for $t<t_{B R}$ the amplitude remains approximately constant. As a matter of fact, one could wonder whether the oscillations of the unstable part could cancel those of the tree level in Eq. (20) so that $f_{\pi}(t)$ remains roughly constant at long times, which would be interpreted as a dissipation effect. Clearly, this is not the case as it can be seen in Figs. 4 and 5. The oscillations coming from $\Delta_{\text {unst }}(t)$ have indeed the same frequency as the tree level ones but their phase is different as it can be seen in Fig. 4. In fact, in that figure one observes that for long times the phase of $\Delta_{\text {unst }}$ is shifted almost $\pi / 2$ with respect to the tree level and therefore the contribution with $\cos M t$ dominates. ${ }^{4}$ In other words, the effect of the pion correlations itself is not enough to make the system equilibrate. This is consistent with the analysis in $[27,37]$ where it is shown that such dis-

\footnotetext{
${ }^{4}$ Working out the expressions for the unstable band given in Appendix $\mathrm{A}$ it can be seen that there are three different terms proportional to the leading long-time exponentials $\exp (q M t / 2)$ in $G_{0}(t, t, k)$. The first one is proportional to $\sin M t$ and changes sign, to leading order in $q$, under a reflection with respect to the band center. The other two are, respectively, time-independent and proportional to $\cos M t$ and they are symmetric under such reflection. Therefore, by integration in $k$, the $\sin M t$ term is suppressed by at least one power of $q$ with respect to the other two. The timeindependent term increases the central value of the oscillations, as seen in Fig. 4.
} 
sipation appears only when the back-reaction is selfconsistently included, which we have not done, as explained before. This issue will be confirmed by the analysis in the next section, where it is shown that the pion number grows exponentially in time even when the loop corrections are included. If the back-reaction was considered, the pion number should reach a maximum value and then stop growing [27]. Our $f(t)$ is an external force whose shape is not changed during the time evolution, which is consistent only below $t_{B R}$. Furthermore, not only the phase of the long-time oscillations is different, but, as it can be seen from Fig. 5, the central value also decreases with time (increases in Fig. 4). This is due to the constant term proportional to $\exp (q M t / 2)$ commented before. We interpret this effect as a reheating of the system [14]. The fact that $f_{\pi}$ decreases with temperature gives support to this idea. Also, assuming that when the system reaches the back-reaction time scale we can use approximately the equilibrium expressions then, according to Eq. (30), an estimate of the final temperature is given by

$$
T_{f}^{2} \simeq 6\left(f_{\pi}^{2}-\overline{f_{\pi}^{s}}\left(t_{f}\right)^{2}\right)
$$

where the bar denotes time average:

$$
\bar{F}\left(t_{f}\right)=\frac{1}{t_{f}} \int_{0}^{t_{f}} d t^{\prime} F\left(t^{\prime}\right) .
$$

For the above estimate of $T_{f}$ it is not important whether we choose $f_{\pi}^{s}$ or $f_{\pi}^{t}$. Thus, for the parameters in case (d) in Figs. 4 and 5 we obtain, for $M t_{f} \simeq 25-30, T_{f} \simeq 125-140$ $\mathrm{MeV}$ which is not far from experimental determinations of the freeze-out temperature [48]. Recall that this value is almost independent of the initial temperature $T_{i}$ and it is therefore compatible with a supercooled initial state. Although our estimate is based on assumptions about the final state, we are using $f_{\pi}$ and not the pion distribution function. The reason is that $f_{\pi}$ always remains close to its equilibrium value, according to our previous discussion, unlike the distribution function which is not thermal, as it will be shown in the next section. We remark that the corrections to $f_{\pi}$ must remain perturbatively small, consistently with the chiral power counting we have discussed in Sec. III and as long as the back reaction effects can be ignored.

Next we will come back to the issue of the pion velocity. To the order we are considering, we have, from Eqs. (35) and (36),

$$
\frac{f_{\pi}^{s}(t)}{f_{\pi}^{t}(t)} \simeq 1+2 L_{12} \frac{q M^{2}}{f_{\pi}^{2}} \sin M t
$$

It is unclear whether one can extrapolate from the analysis in [43] and thus identify the above with the pion velocity $v_{\pi}$. In fact, note that $L_{12}<0$ and then $v_{\pi}>1$ whenever $\sin M t<0$. This is a similar problem as trying to identify $m^{2}(t)$ with a pion mass which, as we have emphasized, is not correct since the chiral symmetry is exactly preserved and the pions remain massless. The problem, as it is discussed below, is trying to define a time-dependent dispersion law, which is meaningless unless additional restrictions are imposed, such as adiabaticity. However, and following our previous argument, by the final time where the equilibrium expressions are meant to be approximately valid, an estimate of the maximum variation of $v_{\pi}$ would be given by taking the time average of the above quantity. Thus we get $\Delta v_{\pi}^{\max }$ $\simeq 0.003$ for $M t_{f}=30$, for the same parameters as before. Note that this correction is even smaller than what is expected from ChPT beyond one loop, namely $\Delta v_{\pi}$ $\simeq 0.14-0.18$ for $T=100 \mathrm{MeV}[44,45]$.

\section{THE PION NUMBER}

\section{A. The nonequilibrium particle number. Definitions}

One should bear in mind that the concept of particle number out of equilibrium is rather subtle. The nature of the problem is well illustrated once more in curved space-time QFT [30]. The particle number depends on the reference frame and thus the initial vacuum state may contain particles during its subsequent time evolution. In other words, the state which is regarded as the vacuum at time $t$ is different from that at $t=0$. However, the particle number can be given a physical meaning in some particular cases. For instance, if the metric is conformally Minkowskian (as the RW spatially flat metric we are considering here) or it is Minkowskian on the space-time boundary. Another interesting regime is when the expansion rate is small compared to the typical frequencies involved, which is the so-called adiabatic limit. Similarly, in nonequilibrium field theory one has to specify the state with respect to which particles are defined. One possibility is to choose the initial Fock space, which is simpler if the initial state is the equilibrium one (the analog of the Minkowski limit). In that case, one considers the time evolution of the initial creation and annihilation operators with the time-dependent density matrix [27,37]. Physically, this corresponds to the number of initial particles. In our case these are massless pions. Since our model preserves the chiral symmetry at all times, pions remain massless for $t>0$. Given the difficulties related to the definition of the nonequilibrium dispersion law (see comments below), we will restrict here to the number of massless pions. Another possibility, when dealing with time-dependent mass terms like the one in Eq. (3) is to define the adiabatic number, where the dispersion law is assumed to be simply $\omega_{k}^{2}(t)=k^{2}+m^{2}(t)$. The particle number is then defined in terms of the creation and annihilation operators that diagonalize the instantaneous Hamiltonian via a Bogoliubov transformation [23]. We remark that the adiabatic limit is consistent for a slowly varying $m^{2}(t)$ and in fact it can be defined only for real $\omega_{k}(t)$. In our case this approximation would be valid only for $k^{2}$ $>q M^{2} / 2$. For small $q$ this would capture anyway the resonance band and hence the parametric amplification. However, as we will see below, it is not even clear whether the dispersion law can be assumed to be adiabatic for all times. Both definitions of particle number coincide at $t=0$.

The nature of our approach makes it more suitable to define the particle number in terms of correlation functions, rather than in the canonical formalism. To illustrate the way we will proceed, let us consider first a free scalar field $\phi$ of 
mass $m$ in thermal equilibrium, so that $n(k)$ is just given by the Bose-Einstein distribution function (12). The timeindependent Hamiltonian of the system is

$$
H=\int d^{3} \vec{x} \frac{1}{2}\left[\dot{\phi}^{2}+(\nabla \phi)^{2}+m^{2} \phi^{2}\right] .
$$

The thermal averaged energy of the system per unit volume is related to the particle number $n(k)$ simply as

$$
\frac{\langle\langle H\rangle\rangle}{V}=\int \frac{d^{3} \vec{k}}{(2 \pi)^{3}} \omega(k)\left[n(k)+\frac{1}{2}\right]
$$

where $k \equiv|\vec{k}|$ and $\omega^{2}(k)=k^{2}+m^{2}$ is the free dispersion law. Thus, from Eq. (42) and the definitions of the two-point functions in Sec. II B,

$$
\begin{aligned}
n(k)+\frac{1}{2}= & \frac{i}{2 \omega(k)}\left[\left.\frac{d}{d t_{1}} \frac{d}{d t_{2}} G_{0}^{>}\left(t_{1}, t_{2}, k\right)\right|_{t_{1}=t_{2}}\right. \\
& \left.+\omega^{2}(k) G_{0}^{>}\left(t_{1}=t_{2}, k\right)\right] \\
= & \frac{i}{2 \omega(k)}\left[-\ddot{G}_{0}^{>}(0, k)+\omega^{2}(k) G_{0}^{>}(0, k)\right]
\end{aligned}
$$

where $G_{0}$ is the free propagator, the dot denotes time derivative and we have used time translation invariance (thermal equilibrium) so that $G_{0}^{>}\left(t_{1}, t_{2}, k\right)=G_{0}^{>}\left(t_{1}-t_{2}, k\right)$, meaning that the particle number is time-independent in equilibrium. Note also that $G_{0}^{>}$above actually stands for $G_{0}^{>11}\left(x, x^{\prime}\right)$ $=\left\langle\phi(x) \phi\left(x^{\prime}\right)\right\rangle$ since we are taking all time arguments in the $C_{1}$ branch in Fig. 1. Using $\left[\partial_{t}^{2}+k^{2}+m^{2}\right] G_{0}^{>}(t, k)=0$ (the equation of motion) we have

$$
n(k)=i \omega(k) G_{0}^{>}(0, k)-\frac{1}{2}=\frac{1}{e^{\beta \omega(k)}-1}
$$

where we have used the solution for the free propagator in equilibrium which can be read off from Eq. (10) with $h_{1}(t, k)$ in Eq. (9), i.e.,

$$
\begin{aligned}
i G_{0}^{>e q}\left(t-t^{\prime}, k\right)= & \frac{1}{2 k}\left\{\left[1+n_{B}(k)\right] e^{-i k\left(t-t^{\prime}\right)}\right. \\
& \left.+n_{B}(k) e^{i k\left(t-t^{\prime}\right)}\right\},
\end{aligned}
$$

Our next step will be to extend the above definitions to the nonequilibrium case. Thus, following the same steps, we will define the nonequilibrium particle number $n(k, t)$ through

$$
\frac{\langle E(t)\rangle}{V}=N \int \frac{d^{3} \vec{k}}{(2 \pi)^{3}} \omega(k, t)\left[n(k, t)+\frac{1}{2}\right]
$$

where $\omega(k, t)$ is (formally) the dispersion law, $\langle E(t)\rangle$ is the total energy of the system and $N$ is the number of particle flavors. In our case $N=3$, the number of different pions. In fact, we should consider a more general definition involving a sum over all internal indices but we will see below that to the order we are considering, all our expressions remain diagonal in isospin space. Thus, the pion density for a given pion type is

$$
\frac{\langle n(t)\rangle}{V}=\int \frac{d^{d-1} k}{(2 \pi)^{d-1}} n(k, t)
$$

where we have kept the space-time dimension $d$ in order to regulate the UV behavior (see below). Several remarks are in order here: As explained before, both the particle number and dispersion law may depend on time. In particular, loops can introduce corrections to the tree level dispersion law. Furthermore, whenever the time dependence appears through the interaction with an external source (as it is the case here) the energy $E(t)$ is not conserved (it is time dependent) as it happens in curved space-time when the back-reaction effects on the metric are ignored. Note that $E(t)$ is the contribution to the energy from the pions only and the oscillations of $f(t)$ transfer energy to the pions making the pion number $n(t)$ grow with time.

In fact, and following once more the curved space-time analogy we have previously discussed, we will calculate the expectation value of the total energy through

$$
\langle E(t)\rangle=\int d^{3} \vec{x} \sqrt{-g}\left\langle T_{0}^{0}(\vec{x}, t)\right\rangle
$$

where $T_{\mu \nu}$ is the energy-momentum tensor defined in terms of the Lagrangian as [35]

$$
T_{\mu \nu}=\frac{2}{\sqrt{-g}} \frac{\delta(\sqrt{-g} \mathcal{L})}{\delta g^{\mu \nu}} .
$$

In Appendix B we have reviewed some useful results regarding the calculation of the classical energy-momentum tensor for the case of interest here (spatially flat RW metric). We remark that, by construction, $T_{\mu \nu}$ is symmetric and classically conserved, i.e., $T_{\mu ; \nu}^{\nu}=0$ where ; denotes the covariant derivative. However, this does not imply necessarily that the energy (46) is time-independent. The reason is that $P_{\mu}$ $=\int d^{3} \vec{x} \sqrt{-g} T_{\mu}^{0}$ is not a covariant four-vector [35]. Note also that in Eq. (46) we are assuming that the expectation value of $T_{\mu \nu}$ has perfect fluid form, which will be the case here (see below).

Therefore, we will proceed by computing the energymomentum tensor to a given order and then calculate the number of particles through Eqs. (44) and (46). This means to deal with expectation values of products of fields at the same space-time point and therefore divergent. We will follow the approach of point-splitting the fields so that the results are written in terms of Green functions. It is important to stress that, as it will be seen below, as long as we use dimensional regularization the final expressions for the particle number are automatically finite, without any need for extra renormalizations of the energy-momentum tensor $[30,49,50]$. 
The simplest example of such point-splitting is provided by our previous derivation of the equilibrium particle number for a scalar theory, where we have replaced for instance

$$
\left\langle\left\langle\dot{\phi}^{2}(\vec{x}, t)\right\rangle\right\rangle=\lim _{\left(\overrightarrow{x^{\prime}}, t^{\prime}\right) \rightarrow(\vec{x}, t)} \frac{d}{d t^{\prime}} \frac{d}{d t} i G^{>}\left(\vec{x}-\vec{x}^{\prime}, t-t^{\prime}\right) .
$$

We will proceed in the same way for the nonequilibrium case. However, as we will see in the next section, sometimes we will have to deal with a field structure in the classical $T_{00}$ which is not symmetric under field exchange, like $\dot{\phi} \phi$. In these cases we will symmetrize first the classical expression and then point-split the fields [30], i.e.,

$$
\begin{aligned}
& \left\langle A_{1}(x) A_{2}(x) \ldots A_{n}(x)\right\rangle \\
& =\frac{1}{n !} \lim _{x_{j} \rightarrow x}\left[\left\langle A_{1}\left(x_{1}\right) A_{2}\left(x_{2}\right) \ldots A_{n}\left(x_{n}\right)\right\rangle\right. \\
& \left.\quad+\left\langle A_{2}\left(x_{2}\right) A_{1}\left(x_{1}\right) \ldots\right\rangle+\cdots\right]
\end{aligned}
$$

where the $x_{j}$ are space-time points, the $A_{j}$ is a shorthand notation to denote either the field or an arbitrary number of its derivatives and the dots denote all possible permutations. Note that we are dealing only with boson fields, which are symmetric under field exchange at different points. As it will become clear below, symmetrizing the fields in this way yields consistent results for the particle number.

Another problem in connection with the point-splitting is the $T_{C}$-ordering of the fields. For instance, we will find fourfield contributions to the energy-momentum tensor and we need to relate them with $T_{C}$-ordered four-point Green functions, so that we can use Wick's theorem to write the result only in terms of two-point functions. We did not have this problem in our previous derivation of the equilibrium particle number, since we were only dealing with products of two fields. Thus, we need to specify how the time arguments of the fields are ordered when taking the $t_{j} \rightarrow t$ limit. We will use the following prescription $[49,50]$

First we will symmetrize over all possible ways of ordering the classical fields, as it is shown in Eq. (49).

Next, for a given ordering of the classical fields with an arbitrary number of field derivatives, we will replace

$$
\begin{aligned}
\left\langle\partial_{\mu_{1}}^{x} \phi(x) \partial_{\mu_{2}}^{x} \phi(x) \phi(x) \ldots\right\rangle & \\
= & \lim _{x_{j} \rightarrow x} * \partial_{\mu_{1}}^{x_{1}} \partial_{\mu_{2}}^{x_{2}} \ldots\left\langle\phi\left(x_{1}\right) \phi\left(x_{2}\right) \phi\left(x_{3}\right) \ldots\right\rangle \\
= & \lim _{x_{j} \rightarrow x} * \partial_{\mu_{1}}^{x_{1}} \partial_{\mu_{2}}^{x_{2}} \ldots\left\langle T \phi\left(x_{1}\right) \phi\left(x_{2}\right) \phi\left(x_{3}\right) \ldots\right\rangle
\end{aligned}
$$

where $\lim _{x_{j} \rightarrow x}^{*}$ means to take the $x_{j} \rightarrow x$ limit keeping the time arguments ordered from left to right, i.e., $t_{1}>t_{2}>\ldots$ (all $t_{j}$ are in the $C_{1}$ branch of the contour in Fig. 1 so that the contour ordering $T_{C}$ becomes the ordinary time-ordering $T$ ). Note that the field derivatives, when present, are pulled out of the $T$-ordering $[49,50]$.

Note that in the second step we are choosing a particular way to take the limit. If such limit exists, the answer should be the same regardless of the order. In this respect it is important to bear in mind that expectation values of products of fields [like $G^{>}\left(x, x^{\prime}\right)$ and its derivatives] have always a welldefined equal-time limit, unlike $T$-ordered products where one has to be careful with ill-defined expressions such as $\delta(0), \delta^{\prime}(0)$ and so on, when taking time derivatives. For instance, suppose that we wanted to use the above prescription with $\langle\dot{\phi} \phi\rangle$ to relate it with $G\left(x_{1}, x_{2}\right)$ $=\left\langle T \phi\left(x_{1}\right) \phi\left(x_{2}\right)\right\rangle$. Then,

$$
\begin{aligned}
\langle\dot{\phi}(t) \phi(t)\rangle & =\frac{1}{2}\langle\dot{\phi}(t) \phi(t)+\phi(t) \dot{\phi}(t)\rangle \\
& =\frac{1}{2} \lim _{t^{\prime} \rightarrow t} *\left(\partial_{t}+\partial_{t}^{\prime}\right) G^{>}\left(t, t^{\prime}\right) \\
& =\frac{1}{2} \lim _{t^{\prime} \rightarrow t} *\left(\partial_{t}+\partial_{t}^{\prime}\right) G\left(t, t^{\prime}\right)
\end{aligned}
$$

where for simplicity we have omitted spatial arguments, which do not play any role here, and lim* means taking the limit so that $t>t^{\prime}$ and then the last step in the above equation holds. Had we taken the limit keeping $t^{\prime}>t$, the answer would have been the same by continuity of $G^{>}\left(t, t^{\prime}\right)$ and its derivatives. In fact, note that this is equivalent to take $t$ and $t^{\prime}$ in $C_{2}$ in Fig. 1 which gives the same answer for $G^{>}$as taking them in $C_{1}$ in the $t^{\prime} \rightarrow t$ limit [33]. Of course, we could have written the result directly in terms of $G^{>}$without specifying the order of the time arguments and the answer is the same either way. In other words, Wick's theorem is trivial for two-point functions. However, for four-point functions the above continuity arguments apply as well and hence this prescription will allow us to use Wick's theorem in the standard way for $T_{C}$-ordered products [4].

\section{B. The number of pions in parametric resonance}

\section{Leading order}

Let us start with the lowest order in ChPT. As explained before, to lowest order it is enough to consider the Lagrangian in Eq. (15) with the RW metric. Furthermore to leading order we only need the $\mathcal{O}\left(\tilde{\pi}^{2}\right)$ terms in that Lagrangian. That is, the "free" Lagrangian given in Eq. (3) for the $\pi(x, t)$ fields. The reason why we can neglect $\mathcal{O}\left(\tilde{\pi}^{4}\right)$ terms to leading order when calculating $\langle E\rangle$ can be understood in terms of Feynman diagrams. What we are doing is starting from diagrams with a given number of vertices of different types and closing them in all possible ways. For instance, at the tree level there is only one point and hence one vertex. Thus, the contribution from the $\mathcal{O}\left(\tilde{\pi}^{2 n}\right)$ is a $n$-loop closed diagram and hence according to Weinberg power counting theorem [15] it contributes as $\mathcal{O}\left(p^{2 n}\right)$ in the chiral power counting. 
Therefore, using the form (15) for the $S_{2}$ action in the parametrization of the $\tilde{\pi}^{a}(\vec{x}, t)$ fields, we have

$$
S_{2}[\tilde{\pi}]=\int_{C} d^{4} x \frac{1}{2} \sqrt{-g} g^{\mu \nu} \partial_{\mu} \tilde{\pi}^{a} \partial_{\nu} \tilde{\pi}^{a}+\mathcal{O}\left(\tilde{\pi}^{4}\right) .
$$

Hence, the energy-momentum tensor (47) to lowest order reads simply

$$
T_{\mu \nu}^{(2,2)}=\partial_{\mu} \tilde{\pi}^{a} \partial_{\nu} \tilde{\pi}^{a}-\frac{1}{2} g_{\mu \nu} \partial^{\alpha} \tilde{\pi}^{a} \partial_{\alpha} \tilde{\pi}^{a}
$$

where we have used Eq. (B4) and the superscript $(n, m)$ in $T_{\mu \nu}$ means a contribution coming from the $S_{n}$ action with $m$ pion fields. The above result is the standard kinetic term in curved space-time. Using the equations of motion to this order $\left(g^{\mu \nu} \partial_{\nu} \tilde{\pi}^{a}\right)_{; \mu}=0$ it is straightforward to check that $T_{\mu \nu}^{(2,2)}$ is covariantly conserved. Besides, from the particular form of $T_{\mu \nu}^{(2,2)}$ above it is not difficult to see that its expectation value has perfect fluid (diagonal) form, that is, $\left\langle T_{i j}\right\rangle$ $=0$ for $i \neq j$ and $\left\langle T_{i 0}\right\rangle=0$, just from spatial translation invariance.

Now, let us consider the above for the RW metric. The total energy defined in Eq. (46) reads, to this order,

$$
\begin{aligned}
\left\langle E^{(2,2)}(t)\right\rangle= & a^{2}(t) \int d^{3} \vec{x} \frac{1}{2}\left\langle\left[\dot{\tilde{\pi}}^{a}(\vec{x}, t)\right]^{2}+\left[\nabla \tilde{\pi}^{a}(\vec{x}, t)\right]^{2}\right\rangle \\
= & \frac{1}{2} \int d^{3} \vec{x}\left\langle\left[\dot{\pi}^{a}\right]^{2}+\left[\nabla \pi^{a}\right]^{2}+\frac{\dot{a}^{2}(t)}{a^{2}(t)}\left[\pi^{a}\right]^{2}\right. \\
& \left.-2 \frac{\dot{a}(t)}{a(t)} \pi^{a} \dot{\pi}^{a}\right\rangle
\end{aligned}
$$

where in the last line we have written the result for the $\pi^{a}(\vec{x}, t)$ fields. The first line above simply states that the energy is conformally equivalent to the Minkowski (equilibrium) result in the $\tilde{\pi}$ parametrization. Recall that the spatially flat RW metric is related to the Minkowski one by a conformal transformation.

Note also that the energy density is not obtained just by replacing $m^{2} \rightarrow m^{2}(t)$ for a free scalar theory, as one could have expected from the Lagrangian (3). That would be equivalent to work in the adiabatic limit and it would have been the answer defining the energy as $H(t)=\int d^{3} \vec{x} \mathcal{H}(\vec{x}, t)$ with the Hamiltonian density $\mathcal{H}=\dot{\pi}(\partial \mathcal{L} / \partial \dot{\pi})-\mathcal{L}$. However, these two definitions are not equivalent in the presence of the external force $a(t)$. In fact it can be checked that adding to the Lagrangian a total derivative $\sqrt{-g} \mathcal{L} \rightarrow \sqrt{-g}\left(\mathcal{L}+A_{; \mu}^{\mu}\right)$ with $A^{\mu}$ a contravariant vector (the action has to remain a scalar) which is a functional of the field and its derivatives, does not change neither the equations of motion nor the energy-momentum tensor defined as Eq. (47). However, $\mathcal{H}$ may change under such transformation. For instance, considering the expression for the action $S_{2}[\pi]$ before integrating by parts to get Eq. (3), i.e, $S_{2}[\pi]=(1 / 2) \int\left[\partial_{\mu} \pi \partial^{\mu} \pi\right.$ $\left.+(\dot{a} / a)^{2} \pi^{2}-2(\dot{a} / a) \dot{\pi} \pi\right]$, one gets $\mathcal{H}=(1 / 2)\left[\dot{\pi}^{2}+(\nabla \pi)^{2}\right.$ $\left.-(\dot{a} / a)^{2} \pi^{2}\right]$. Therefore in that case the "mass" term would be proportional to $-(\dot{a} / a)^{2}$ rather than to $-\ddot{a} / a$. In this case $A^{0}=(\dot{a} / a) \pi^{2}$. As we have said before, none of these expressions for the energy density is time-independent.

Nevertheless, there is a way to check the consistency of the above result which in fact gives us a hint of how to include the back-reaction effects. If we let $a(t)$ [or $f(t)]$ be a classical field (independent of $\vec{x}$ for simplicity) in the Lagrangian, then, by considering also its equation of motion, it can be checked that $\left\langle\dot{E}^{(2,2)}(t)\right\rangle=0$. Moreover, $H(t)$ coincides with Eq. (52) when the extra term coming from $\mathcal{H}$ $\rightarrow \mathcal{H}+\dot{a}(\partial \mathcal{L})(\partial \dot{a})$ is considered. From this point of view, in the present approach we are just considering that the field $a$ does not receive quantum corrections and its equation of motion is dominated by the kinetic term, which depends only on $g_{\mu \nu}$ but not on the pion fields. That term is nothing but the counterpart of the Einstein-Hilbert action, yielding the Einstein equation for $g_{\mu \nu}$ when no matter fields are present [35].

At this point let us recall that we are considering only the leading order in $q$ consistently with our power counting. Therefore we can neglect the $\dot{a}^{2}$ term in Eq. (52). Now, let us apply our point-splitting prescription to the remaining terms in Eq. (52). We will write the result in terms of $h_{1}(t)$ in Eq. (10). Let us consider first the term proportional to $\dot{a}$. According to our previous discussion,

$$
\begin{aligned}
\left\langle\pi^{a}(x) \dot{\pi}^{a}(x)\right\rangle & =3 i \lim _{t^{\prime} \rightarrow t} \int \frac{d^{3} \vec{k}}{(2 \pi)^{3}} \frac{1}{2}\left[\frac{d}{d t}+\frac{d}{d t^{\prime}}\right] G_{0}^{>}\left(t, t^{\prime}, k\right) \\
& =\frac{3}{2} \int \frac{d^{3} \vec{k}}{(2 \pi)^{3}}\left[1+2 n_{B}(k)\right] \frac{d}{d t} \mid h_{1}\left(t,\left.k\right|^{2}\right. \\
& =\frac{3 i}{2} \dot{G}_{0}(t)
\end{aligned}
$$

where we have used Eqs. (10) and (14). The above result deserves some comments: first, we realize that if we had not taken the symmetric limit, the answer would have been complex in general, whereas it is manifestly real when the two contributions are added together. This is a consistency check of our point-splitting prescription. Second, since $h_{1}(t, k)$ $=i \exp (-i k t) / \sqrt{2 k}+\mathcal{O}(q)$ (the leading order is the equilibrium solution) then $\left\langle\pi^{a} \dot{\pi}^{a}\right\rangle=\mathcal{O}(q)$. In other words, for the time scales we are considering (below $t_{B R}$ ) $G_{0}(t) / f_{\pi}^{2}$ $=\mathcal{O}(q)$. Therefore and since $\dot{a}=\mathcal{O}(q)$ as well, to leading order we just have

$$
\begin{aligned}
\frac{\left\langle E^{(2,2)}(t)\right\rangle}{V}= & \frac{3}{2} \int \frac{d^{3} \vec{k}}{(2 \pi)^{3}}\left\{\left[1+2 n_{B}(k)\right]\right. \\
& \left.\times\left[\left|\dot{h}_{1}(t, k)\right|^{2}+k^{2}\left|h_{1}(t, k)\right|^{2}\right]\right\}+\mathcal{O}\left(q^{2}\right) .
\end{aligned}
$$

Hence, according to Eq. (44), we find that the number of massless pions of a given type is given to leading order by 


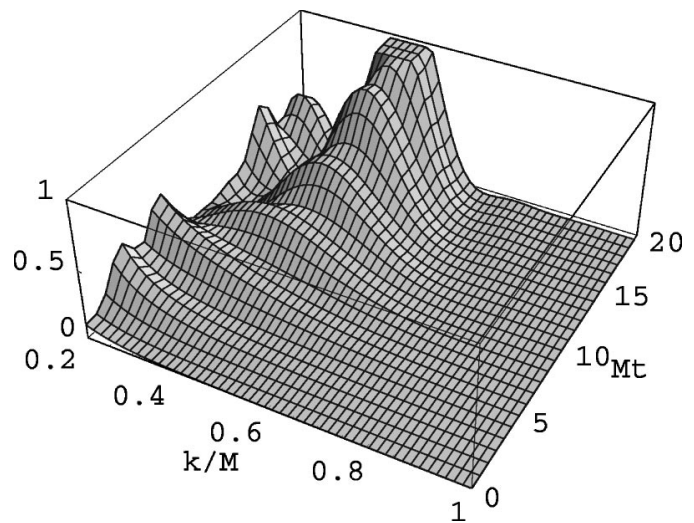

FIG. 6. $n(k, t)$ for $M=600 \mathrm{MeV}, T_{i}=50 \mathrm{MeV}$, and $q=0.2$.

$\left.n^{L O}(k, t)=\frac{1}{2 k}\left|\dot{h}_{1}(t, k)\right|^{2}+k^{2}\left|h_{1}(t, k)\right|^{2}\right]\left[1+2 n_{B}(k)\right]-\frac{1}{2}$.

This expression coincides with the one given in [27,24] in the canonical operator formalism. Note that to this order the answer for the adiabatic number of pions would have been the same, since $m^{2}(t)=\mathcal{O}(q)$. Note also that our initial conditions (9) imply that $n(k, 0)=n_{B}(k)$ as it should.

So far we have considered the general result in ChPT, for arbitrary $f(t)$. Let us now particularize to the parametric resonance case in Eq. (20). First, in order to analyze the UV behavior of the pion density in Eq. (45), we need the solution $h_{1}$ to the Mathieu equation in the stable band (large $k$ ). Using the approximate solutions to leading order in $q$ (see Appendix A) one has

$$
\begin{aligned}
& \left|h_{1}(t, k)\right|^{2}=\frac{1}{2 k}\left\{1+\frac{q M^{2}}{4} \frac{\sin M t-\frac{M}{2 k} \sin 2 k t}{k^{2}-M^{2} / 4}+\mathcal{O}\left(q^{2}\right)\right\} \\
& \left|\dot{h}_{1}(t, k)\right|^{2}=\frac{k}{2}\left\{1-\frac{q M^{2}}{4} \frac{\sin M t-\frac{M}{2 k} \sin 2 k t}{k^{2}-M^{2} / 4}+\mathcal{O}\left(q^{2}\right)\right\}
\end{aligned}
$$

for $k>k_{0}$ (see Sec. III). Thus, we see that the possible logarithmic divergence in $d \rightarrow 4$ [i.e., that coming from the term which is not proportional to $n_{B}(k)$ in Eq. (55)] cancels exactly to leading order in $q$ and therefore the total number is finite. This justifies our approach of point-splitting the fields as long as we remain within the DR scheme.

The pion number will grow exponentially in time due to the contribution of the unstable band. By the same arguments as those used for the two-point function, $n(k, t)$ will grow in time typically with $\exp (q M t / 2)$. In Fig. 6 we have plotted $n(k, t)$ for the choice of parameters (d) in Fig. 4. We observe that the pion number grows to order one within the resonance band and before the back-reaction time. We have also plotted the time average of the pion distribution function $n(k, t)$ in Fig. 7, taking $M t_{f}=30$ (see our comments in Sec. IV C).

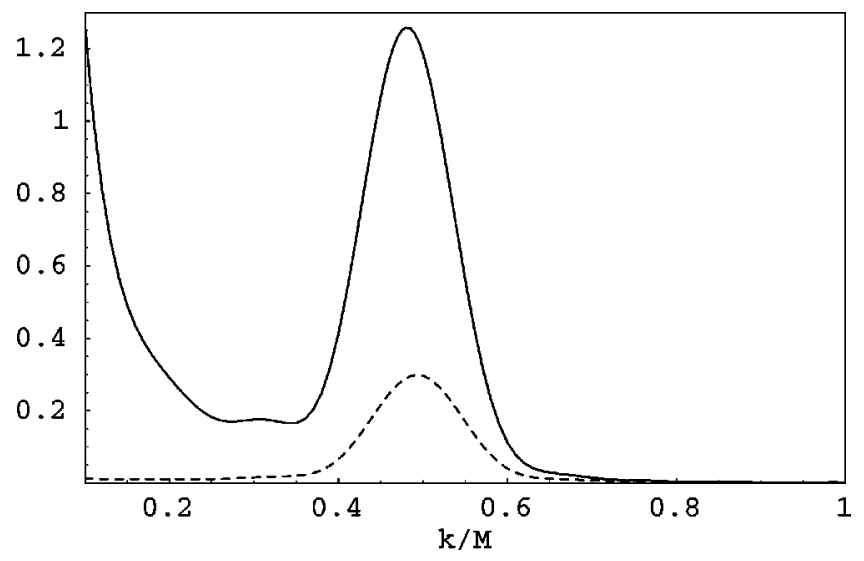

FIG. 7. The solid line is the averaged pion number $\bar{n}\left(k, t_{f}\right)$ for $t_{f}=30 / M$ and the dashed line is $\left(k^{2} / M^{2}\right) \bar{n}\left(k, t_{f}\right)$. Here, $T_{i}=50 \mathrm{MeV}, q=0.2$ and $M=600 \mathrm{MeV}$.

We observe the typical peak of parametric resonance amplification at $k \simeq 300 \mathrm{MeV}$, which would be seen in the final pion spectra [25] although single pion distributions might be not enough to disentangle from states which are not DCClike and one needs to consider higher order pion correlation functions $[9,10]$. The width of the resonance band is $\Delta k$ $\simeq q M / 2=0.1 M$ for this case. Note also that the final shape is very different from a thermal Bose-Einstein distribution function, although both diverge for $k \rightarrow 0$, which reflects Bose-Einstein condensation for massless particles. The physical pion number density in momentum space is $k^{2} n(k, t)$, whose time average we have also plotted in Fig. 7 (dashed line). Note that decreasing the value of $M$ would make the Bose-Einstein contribution $n_{B}(k)$ in Eq. (55) become more important for small $k$ in Fig. 7 (solid line) since $T_{i} / M$ increases. The resonant behavior around $k=M / 2$ does not change with $M$, although the final time $t_{f}$ does, as discussed in Sec. IV. Finally, note the strong decreasing of the pion number for large $k$ in Fig. 7, as expected from our previous comments about the UV behavior.

Finally, the pion density (45) as a function of time is shown in Fig. 8. Our results agree numerically with the predictions of the $O(4)$ model in the spinodal regime [23,22]. As we have previously commented, the particle number

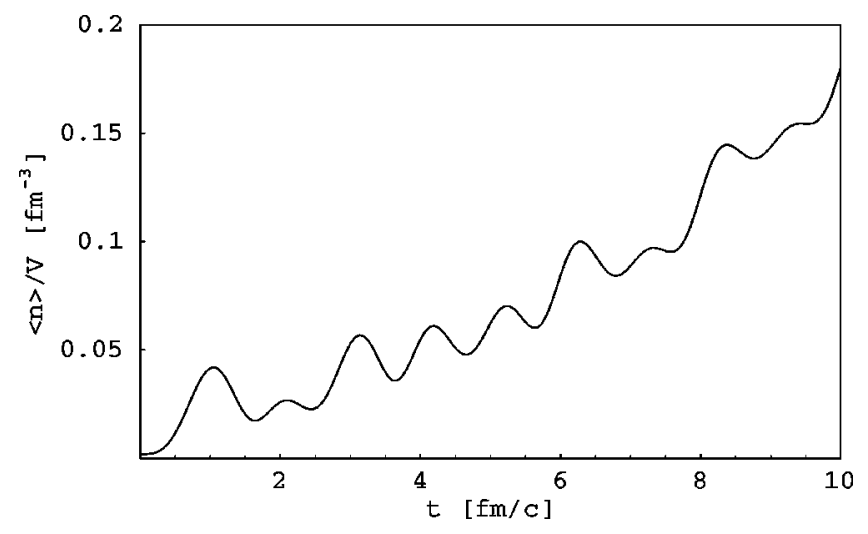

FIG. 8. The pion density for $T_{i}=50 \mathrm{MeV}, q=0.2$ and $M=600 \mathrm{MeV}$. 
grows in time without stop. One may wonder whether the one-loop NLO effects may change this picture, but this is not the case, as we are going to see in the next section. The origin of this behavior, as emphasized before, is that we have not taken into account the back-reaction properly [27]. As long as we remain below the back-reaction time, our results for the pion number can be trusted.

\section{Next to leading order}

There are three types of NLO contributions to the pion number:

(1) The one-loop corrections to the two-point function, which we have already discussed in Sec. IV A [diagrams (a) and (b) in Fig. 3].

(2) The contribution of the $\mathcal{O}\left(p^{4}\right)$ Lagrangian (16) to the energy-momentum tensor. According to our previous discussion, only two-field terms contribute to NLO, namely $\left\langle E^{(4,2)}\right\rangle$ in our notation.

(3) Four-field terms in the $\mathcal{O}\left(p^{2}\right)$ Lagrangian, i.e., $\left\langle E^{(2,4)}\right\rangle$.

Before proceeding, we should insist that, as commented before, we are considering the number of massless pions, ignoring possible one-loop modifications of the dispersion law. We must stress that the difficulties associated to the definition of the time-dependent dispersion law are similar to those related to the nonequilibrium particle number. Physically it makes sense to define it asymptotically at long times but it is not clear whether one can actually define an instantaneous dispersion law, unless one follows the adiabatic approximation. Nonetheless, we believe that such corrections would not change qualitatively the time evolution of the pion number. In Sec. IV we have already commented on the inmedium dispersion law and its relationship with the pion decay constants $f_{\pi}^{s, t}$. Remember that in equilibrium the pion dispersion law remains unchanged to one-loop in ChPT. While a detailed nonequilibrium extension of the results in [43] is out of the scope of this work, qualitatively we expect a similar one-loop behavior in our case with perhaps a change in the pion velocity of order $\left|f_{\pi}^{s}(t) / f_{\pi}^{t}(t)\right|$ as Eq. (41) shows. That contribution is proportional to $L_{12}$ and bounded in time. In any case, if we write $\omega=k+\Sigma(k, t)$ with $\Sigma(k, t)=\mathcal{O}(q)$ a bounded function in time, the contribution to the pion number (44) would be $n \rightarrow n-[\Sigma(k, t) / k] n_{B}(k)$. This is a bounded correction that does not change the relevant features of pion production we are analyzing here.

Let us then start with the NLO corrections of type 1 . According to our power counting, this correction affects only the terms in $E^{(2,2)}$. The NLO correction to the pion two-point function has been calculated in [28] as explained before. Let us write it as $G^{>}\left(t, t^{\prime}, k\right)=G_{0}^{>}\left(t, t^{\prime}, k\right)+\Delta\left(t, t^{\prime}, k\right)$. Then, according to our arguments in the previous section, the contribution of this correction to the pion number is given to $\mathcal{O}(q)$ by

$$
n(k, t) \rightarrow n(k, t)+\frac{i}{2 k}\left[\left.\frac{d}{d t} \frac{d}{d t^{\prime}} \Delta\left(t, t^{\prime}, k\right)\right|_{t=t^{\prime}}+k^{2} \Delta(t, t, k)\right] .
$$

Now, from the results in [28] [Eqs. (15)-(17) in that paper $^{5}$ ] we obtain:

$$
\begin{aligned}
& i\left[\left.\partial_{t} \partial_{t^{\prime}} \Delta\left(t, t^{\prime}, k\right)\right|_{t=t^{\prime}}+k^{2} \Delta(t, t, k)\right] \\
& =-i f_{1}(t) k^{2} G_{0}^{>}(t, t)-\left.\frac{i}{2} \dot{f}_{1}(t)\left[\partial_{t}+\partial_{t^{\prime}}\right] G_{0}^{>}\left(t, t^{\prime}\right)\right|_{t=t^{\prime}}+\left.\left[2 \Delta_{2}(t)-i f_{1}(t)\right] \partial_{t} \partial_{t^{\prime}} G_{0}^{>}\left(t, t^{\prime}\right)\right|_{t=t^{\prime}} \\
& \quad+\int_{0}^{t} d u \Delta_{1}(u, k)\left\{\left[\left(\partial_{t} G_{0}^{>}(t, u)\right)^{2}-\left(\partial_{t} G_{0}^{>}(u, t)\right)^{2}\right]+k^{2}\left[\left(G_{0}^{>}(t, u)\right)^{2}-\left(G_{0}^{>}(u, t)\right)^{2}\right]\right\} \\
& \quad+\int_{0}^{t} d u \Delta_{2}(u)\left\{\left[\left(\partial_{u} \partial_{t} G_{0}^{>}(t, u)\right)^{2}-\left(\partial_{u} \partial_{t} G_{0}^{>}(u, t)\right)^{2}\right]+k^{2}\left[\left(\partial_{u} G_{0}^{>}(t, u)\right)^{2}-\left(\partial_{u} G_{0}^{>}(u, t)\right)^{2}\right]\right\} .
\end{aligned}
$$

Here, the $k$-dependence of $G_{0}^{>}\left(t, t^{\prime}, k\right)$ has been suppressed for simplicity, $f_{1}(t)$ is the function in Eq. (19) appearing in the $\mathcal{O}\left(p^{4}\right)$ Lagrangian and we have used that $\partial_{t}\left[G_{0}^{>}\left(t, t^{\prime}\right)-G_{0}^{<}\left(t, t^{\prime}\right)\right]=-1$. The explicit form of the functions $\Delta_{1}(t, k)$ and $\Delta_{2}(t)$ is given in [28]. What is important for our purposes here is that $\Delta_{1,2}=\mathcal{O}(q)$ within the range of validity of our approximation, i.e., while $G_{0}(t) / f_{\pi}^{2}=\mathcal{O}(q)$. In fact, $\Delta_{2}(t)=G_{0}(t) / f^{2}(t)$, while the precise form of $\Delta_{1}$ is unimportant here (see below). Hence, to $\mathcal{O}(q)$ it is enough to replace in Eq. (56) the leading orderterm in $q$ for $G_{0}^{>}$, which is nothing but the equilibrium so- lution (43). ${ }^{6}$ This simplifies considerably the above expression. In fact, the dependence with $\Delta_{1}(t, k)$ disappears and Eq. (56) reduces to

\footnotetext{
${ }^{5}$ There are two misprints in Eq. (16) in [28]. The term $6 \ddot{f}(\tilde{t}) / f(\tilde{t})$ multiplying $G_{0}(\tilde{t})$ should $\operatorname{read} 4 \ddot{f}(\tilde{t}) / f(\tilde{t})$ and the term $-2 \ddot{G}_{0}(\tilde{t})$ should read $-\ddot{G}_{0}(\tilde{t})$. None of them affects the results here, since they are included in the $\Delta_{1}(t, k)$ function in Eq. (56).

${ }^{6}$ This holds also in the unstable band without expanding in $q$ in the leading exponentials $\exp \mu M t$ where $\mu=\mathcal{O}(q)$ is the Floquet characteristic exponent (see Appendix A).
} 


$$
\begin{array}{r}
i\left[\left.\frac{d}{d t} \frac{d}{d t^{\prime}} \Delta\left(t, t^{\prime}, k\right)\right|_{t=t^{\prime}}+k^{2} \Delta(t, t, k)\right] \\
\quad=-k\left[1+2 n_{B}(k)\right]\left[f_{1}(t)+i \frac{G_{0}(t)}{f_{\pi}^{2}}\right]+\mathcal{O}\left(q^{2}\right) .
\end{array}
$$

Therefore, the correction to the pion number from this part is, neglecting $\mathcal{O}\left(q^{2}\right)$,

$$
n(k, t) \rightarrow n(k, t)-\frac{1}{2}\left[1+2 n_{B}(k)\right]\left[f_{1}(t)+i \frac{G_{0}(t)}{f_{\pi}^{2}}\right]
$$

where $f_{1}(t)=-12\left(2 L_{11}+L_{12}\right) \ddot{f}(t) / f_{\pi}^{3}+\mathcal{O}\left(q^{2}\right)$.

Note that in our one-loop calculation of $f_{\pi}$ in Sec. IV we have shown that the combination $-12 L_{11} \ddot{f} / f-i G_{0}(t)$ is finite. This is the combination appearing in Eqs. (32),(33) with $f_{1}(t)$ and $f_{2}(t)$ in Eq. (19). However we have here $-24 L_{11} \ddot{f} / f+i G_{0}(t)$, which diverges. The only possible way out is then that the remaining NLO corrections (types 2 and 3) combine with this one in such a way that the answer for the pion number is finite.

Let us then consider type 2 corrections, i.e, those coming from the energy-momentum tensor to fourth order in derivatives. By the same argument as before, only two-field terms contribute to this order. Hence, from the Lagrangian in Eq. (16) we can concentrate only in the $L_{11}$ and $L_{12}$ terms. The energy-momentum tensor coming from that Lagrangian is calculated in Appendix B. The final expression for $T_{\mu \nu}^{(4,2)}$ is displayed in Eq. (B7) for arbitrary metric and the contribution to the energy for the RW metric is given in Eq. (B9). Note that we could have used the equations of motion to second order to simplify some of the terms in Eq. (B9), writing for instance $\ddot{\pi}$ in terms of $\Delta \pi$ and $\pi^{2}$ and so on. It is clear that to $\mathcal{O}(q)$ we only need to consider the terms proportional to $g_{1}, g_{5}$ and $g_{7}$ in that expression. We have

$$
\begin{aligned}
& \left\langle\partial_{i} \pi^{a}(\vec{x}, t) \partial_{i} \dot{\pi}^{a}(\vec{x}, t)\right\rangle \\
& \quad=\frac{3 i}{2} \lim _{t^{\prime} \rightarrow t} \int \frac{d^{3} \vec{k}}{(2 \pi)^{3}} k^{2}\left[\frac{d}{d t}+\frac{d}{d t^{\prime}}\right] G_{0}^{>}\left(t, t^{\prime}, k\right)
\end{aligned}
$$

and

$$
\begin{aligned}
\left\langle\dot{\pi}^{a}(\vec{x}, t) \ddot{\pi}^{a}(\vec{x}, t)\right\rangle= & \frac{3 i}{2} \lim _{t^{\prime} \rightarrow t} \int \frac{d^{3} \vec{k}}{(2 \pi)^{3}} \frac{d}{d t} \frac{d}{d t^{\prime}}\left[\frac{d}{d t}+\frac{d}{d t^{\prime}}\right] \\
& \times G_{0}^{>}\left(t, t^{\prime}, k\right)
\end{aligned}
$$

where we have retained only the LO propagator $G_{0}^{>}$since the two terms above are multiplied by $g_{5}(t)$ and $g_{7}(t)$ which are already $\mathcal{O}(q)$. Now, since $G_{0}^{>}\left(t, t^{\prime}, k\right)=G_{0}^{>e q}\left(t-t^{\prime}, k\right)$ $+\mathcal{O}(q)$ with $G_{0}^{>e q}\left(t-t^{\prime}, k\right)$ in Eq. (43) it is clear that the contributions (59), (60) are both $\mathcal{O}(q)$ and therefore they do not contribute to the order we are considering here.

Hence, the only correction of this kind is the one given by the term proportional to $g_{1}(t)$ in Eq. (B9). This term is of the form already analyzed in Sec. V B 1. From our results there, we have that the correction to the particle number from $\left\langle E^{(4,2)}\right\rangle$ is then given to $\mathcal{O}(q)$ by

$$
n(k, t) \rightarrow n(k, t)+\left[1+2 n_{B}(k)\right] \frac{2 g_{1}(t)}{f_{\pi}^{3}}
$$

where $g_{1}(t)=-3\left(2 L_{11}+L_{12}\right) \ddot{f}(t)+\mathcal{O}\left(q^{2}\right)$.

Finally, we shall consider the contribution of four fields from the $\mathcal{O}\left(p^{2}\right)$ Lagrangian in Eq. (15) to the energy momentum tensor. Expanding to $\mathcal{O}\left(\tilde{\pi}^{4}\right)$ in that Lagrangian we find

$$
T_{00}^{(2,4)}=\frac{f^{2}(t)}{2 f^{4}} \sum_{a, b=1}^{3} \tilde{\pi}_{a} \tilde{\pi}_{b}\left[\dot{\tilde{\pi}}_{a} \dot{\tilde{\pi}}_{b}+\left(\nabla \tilde{\pi}_{a}\right)\left(\nabla \tilde{\pi}_{b}\right)\right]
$$

Now we should write the energy (46) to this order for the $\pi^{a}=\tilde{\pi}^{a} f(t) / f$ fields, from the above expression. However, according to our power counting, since this contribution to the energy is $\mathcal{O}\left(p^{2}\right)$ with respect to the tree level, it must be at least $\mathcal{O}(q)$. This will be confirmed by our subsequent calculation. Therefore, when changing from the $\tilde{\pi}$ fields to the $\pi$ fields we can simply ignore the terms proportional to $\dot{f}(t)$. Hence, to this order it is enough to replace simply $\tilde{\pi}$ by $\pi$ and $f(t)$ by $f$ in Eq. (62) and therefore

$$
\begin{aligned}
E^{(2,4)}= & \frac{1}{2 f^{2}} \int d^{3} \vec{x}\left\{\sum _ { a , b = 1 } ^ { 3 } \pi _ { a } ( \vec { x } , t ) \pi _ { b } ( \vec { x } , t ) \left[\dot{\pi}_{a}(\vec{x}, t) \dot{\pi}_{b}(\vec{x}, t)\right.\right. \\
& \left.\left.+\left(\nabla \pi_{a}(\vec{x}, t)\right)\left(\nabla \pi_{b}(\vec{x}, t)\right)\right]\right\} .
\end{aligned}
$$

We have to calculate now the expectation value of the above quantity. Note that this is the first time where we find the problem of the $T$-ordering discussed in Sec. V A, apart from the issue of the symmetrization of the classical fields. We will follow the prescription explained in that section for the different field structures appearing in Eq. (63). Let us consider first the terms $\left\langle\pi_{a} \pi_{b} \partial_{\alpha} \pi_{a} \partial_{\alpha} \pi_{b}\right\rangle$ with $a \neq b$ and where the Lorenz index $\alpha$ is fixed, i.e., is not summed over. We have

$$
\begin{aligned}
\left\langle\pi_{a}(x)\right. & \left.\pi_{b}(x) \partial_{\alpha} \pi_{a}(x) \partial_{\alpha} \pi_{b}(x)\right\rangle \\
= & \frac{1}{4 !} \lim _{x_{j} \rightarrow x}^{*}\left[\partial _ { \alpha } ^ { x _ { 3 } } \partial _ { \alpha } ^ { x _ { 4 } } \left\langleT \pi_{a}\left(x_{1}\right) \pi_{b}\left(x_{2}\right)\right.\right. \\
& \left.\left.\times \pi_{a}\left(x_{3}\right) \pi_{b}\left(x_{4}\right)\right\rangle+\cdots\right]
\end{aligned}
$$


where the dots stand for all the permutations of the four fields in the expectation value. Now, using Wick's theorem,

$$
\begin{aligned}
& \left\langle T \pi_{a}\left(x_{1}\right) \pi_{b}\left(x_{2}\right) \pi_{a}\left(x_{3}\right) \pi_{b}\left(x_{4}\right)\right\rangle \\
& \quad=-G_{0}\left(x_{1}, x_{3}\right) G_{0}\left(x_{2}, x_{4}\right)\left[1+\mathcal{O}\left(p^{2} / \Lambda_{\chi}^{2}\right)\right] \text { for } a \neq b
\end{aligned}
$$

which is the only order we have to retain according to our power counting (remember that this contribution to the energy is already NLO and therefore it is enough to keep the LO propagator) and we have taken into account that the lowest order action in Eq. (3) is diagonal in isospin space, so that to lowest order the $T$-product of fields with different indices factorizes. Note also that the result to this order is independent of the $a, b$ indices as long as $a \neq b$. Collecting the different permutations we find

$$
\begin{aligned}
& \left\langle\pi_{a}(x) \pi_{b}(x) \partial_{\alpha} \pi_{a}(x) \partial_{\alpha} \pi_{b}(x)\right\rangle \\
& \quad=-\left[\left.\frac{1}{2}\left(\partial_{\alpha}^{x}+\partial_{\alpha}^{x^{\prime}}\right) G_{0}^{>}\left(x, x^{\prime}\right)\right|_{x=x^{\prime}}\right]^{2} \quad(a \neq b) .
\end{aligned}
$$

It is clear, following the same arguments as before, that the above quantity is $\mathcal{O}\left(q^{2}\right)$ since $\left(\partial_{\alpha}^{x}+\partial_{\alpha}^{x^{\prime}}\right) G_{0}^{>}\left(x, x^{\prime}\right)$ $=\mathcal{O}(q)$. Thus, we only need to consider the terms with $a$ $=b$ in Eq. (63) to $\mathcal{O}(q)$. For a given $\pi$ field and $\alpha$ fixed,

$$
\begin{aligned}
\left\langle\pi^{2}(x)\left[\partial_{\alpha} \pi(x)\right]^{2}\right\rangle= & \frac{1}{6} \lim _{x_{j} \rightarrow x}^{*}\left(\partial_{\alpha}^{x_{1}} \partial_{\alpha}^{x_{2}}+\partial_{\alpha}^{x_{1}} \partial_{\alpha}^{x_{3}}+\cdots\right) \\
& \times\left\langle T \pi\left(x_{1}\right) \pi\left(x_{2}\right) \pi\left(x_{3}\right) \pi\left(x_{4}\right)\right\rangle .
\end{aligned}
$$

Thus, Wick's theorem gives now

$$
\begin{aligned}
\left\langle T \pi\left(x_{1}\right)\right. & \left.\pi\left(x_{2}\right) \pi\left(x_{3}\right) \pi\left(x_{4}\right)\right\rangle \\
= & -\left[G_{0}\left(x_{1}, x_{2}\right) G_{0}\left(x_{3}, x_{4}\right)+G_{0}\left(x_{1}, x_{3}\right) G_{0}\left(x_{2}, x_{4}\right)\right. \\
& \left.+G_{0}\left(x_{1}, x_{4}\right) G_{0}\left(x_{2}, x_{3}\right)\right] .
\end{aligned}
$$

Now, we take the Fourier transform in the spatial components of the above expression. Our prescription for the $\lim ^{*} x_{j} \rightarrow x$ is equivalent to replace all the $G_{0}$ above by $G_{0}^{>}$ (see our comments in Sec. V A). Thus, taking into account once more that $G_{0}^{>}\left(t, t^{\prime}, k\right)=G_{0}^{>e q}\left(t-t^{\prime}, k\right)+\mathcal{O}(q)$, $G_{0}^{>}(t, t, k)=G_{0}(t, t, k)$ and $G_{0}(t) / f^{2}=\mathcal{O}(q)$, we find for the combination appearing in Eq. (63),

$$
\begin{aligned}
& \left\langle\pi^{2}(\vec{x}, t)\left\{[\dot{\pi}(\vec{x}, t)]^{2}+[\nabla \pi(\vec{x}, t)]^{2}\right\}\right\rangle \\
& \quad=i G_{0}(t) \int \frac{d^{d-1} \vec{k}}{(2 \pi)^{d-1}} k\left[1+2 n_{B}(k)\right]+\mathcal{O}\left(q^{2}\right) .
\end{aligned}
$$

Therefore, from Eq. (63) we find that the contribution of the corrections of type V B 2 to the particle number is given by

$$
n(k, t) \rightarrow n(k, t)+\left[1+2 n_{B}(k)\right] \frac{i G_{0}(t)}{2 f_{\pi}^{2}} .
$$

Finally, collecting the contributions to the pion number to NLO, namely, Eqs. (58), (61) and (65) we find an interesting result: the total NLO correction to $\mathcal{O}(q)$ vanishes, i.e.,

$$
n^{N L O}(k, t)=n^{L O}(k, t)+\mathcal{O}\left(q^{2}\right)
$$

with $n^{L O}(k, t)$ in Eq. (55).

Note the completely different origin of the three NLO contributions and remember that each of them was UV divergent so that there were only two alternatives: either they cancel or they appear in the combination (32),(33). Thus, the only NLO correction to the pion number is due to the change in the dispersion law. Remember that the result (66) holds for massless pions. Note that, in turn, we have shown the absence of NLO corrections to the pion number for equilibrium ChPT. This is indeed consistent since we know that in equilibrium in the chiral limit the pion dispersion law is unchanged to one loop in ChPT. Therefore, the pion distribution function has to be the Bose-Einstein one for massless particles since the system remains in thermal equilibrium.

Thus, the numerical results showed in Figs. 6 and 7 remain valid to NLO. As commented before, we did not expect that the NLO corrections make the pion number stop growing since we have not included the back reaction and the energy $E(t)$ to this order is still not conserved. Our results are valid below the back reaction time and should account for all the relevant pion production.

\section{CONCLUSIONS AND OUTLOOK}

Chiral perturbation theory can be used to describe nonequilibrium phenomena. In particular, in this work we have showed that pion production can be accommodated in ChPT in the parametric resonance regime. The physical situation where this analysis is meant to be useful is the late time expansion of the plasma formed after a relativistic heavy ion collision. Pion production is important in the context of hadronization and production of disoriented chiral condensates during the chiral phase transition.

In the present approach, we have considered the nonlinear sigma model in the chiral limit, where the pion decay constant is time-dependent. This is a nonequilibrium effective model with a well-defined perturbative expansion and power counting near equilibrium. Besides, using the analogy of this model with curved space-time QFT, we have been able to construct the fourth order Lagrangian and implement renormalization in a consistent fashion. The parametric resonance regime corresponds to take $f_{\pi}(t)$ oscillating around its equilibrium position. To lowest order $f_{\pi}(t)$ corresponds to the vacuum expectation value of the $\sigma$ field in the $O(4)$ model. Thus, the pion equation of motion to lowest order in the amplitude oscillations becomes a Mathieu equation, which has resonance bands in momentum space. The pion correlator grows exponentially in time, yielding explosive pion production. This approximation is consistent until the time when the back reaction effects due to the pion correlations become important. We have estimated this time scale for different choices of the initial values of the amplitude, frequency and temperature. 
The two observables we have analyzed here are the pion decay constants and the pion number up to one loop in ChPT. Our main results are the following. For $f_{\pi}(t)$ the nonequilibrium corrections are basically of oscillatory nature until the back reaction time. However, the central value tends to decrease, which can be interpreted in terms of a reheating of the system. Besides, a small difference between $f_{\pi}^{s}$ and $f_{\pi}^{t}$ is induced, unlike the equilibrium case where it vanishes at one loop. Using the equilibrium result, we have estimated the final temperature and the averaged value of $f_{\pi}^{s}-f_{\pi}^{t}$ which is related to the in-medium pion velocity in equilibrium.

As for the particle number, we have first introduced a suitable definition in terms of the energy-momentum tensor and Green functions. We have showed that in dimensional regularization there is no need for extra renormalizations and we can use a point-splitting prescription consistently. The number of initial particles at tree level coincides basically with the result in the $O(4)$ model. At one loop we have found that all the relevant contributions cancel, to leading order in the oscillations amplitude. This result holds also in equilibrium, which is a particular case of this analysis. Thus, our prediction for the particle number is just the tree level result, which gives pion exponential growth in time. We have given numerical results both for the pion distribution function $n(k, t)$ and for the pion density $\langle n(t)\rangle / V$. The final distribution function which would be observed has the typical peak of parametric resonance at the center of the unstable band $(k \simeq M / 2)$.

The reason why $f_{\pi}(t)$ and $\langle n(t)\rangle / V$ are not damped is because we have not taken into account the back-reaction effects which would change the original ansatz for the pion decay constant. When taken into account, those effects should make the particle number stop growing and give energy conservation. Nevertheless, our approach is perfectly valid until the time where these dissipation effects are important and therefore we believe we capture the essential behavior concerning explosive pion production.

We must stress than, apart from being a physically interesting case, the example analyzed here has allowed us to show explicitly the renormalization of our model to one loop, which is not trivial because of the presence of new nonequilibrium infinities. In fact, we believe that our methods could be useful for other nonequilibrium field theoretical models.

There are many directions in which this work can be extended. Perhaps the most important would be to be able to include the above mentioned back-reaction effects, in order to understand dissipation properly. Other extensions include to consider nonzero physical pion masses and other relevant observables such as the correlation length or higher pion correlators, which are important to clarify the issue of DCC formation and to obtain predictions testable in RHIC. In addition, photon production can be studied by gauging the NLSM, including the Wess-Zumino-Witten term, responsible for the anomalous decay $\pi^{0} \rightarrow \gamma \gamma$. Work along these lines is in progress.

\section{ACKNOWLEDGMENTS}

The author wishes to thank A.L. Maroto for useful comments and discussions. Financial support from CICYT,
Spain, projects AEN97-1693 and FPA2000-0956 is acknowledged.

\section{APPENDIX A: SOLUTIONS OF THE MATHIEU EQUATION}

Here we will summarize the main results used in the text concerning the solutions of Mathieu differential equation. All these results can be found in $[51,52]$.

According to Floquet's theorem, there is always a solution of the Mathieu equation (21) of the form

$$
F_{\nu}(z)=e^{i \nu z} P(z)
$$

where $P(z)$ is a periodic function with period $\pi$ and $\nu$ is called the characteristic exponent, which depends on $a$ and $q$ and it plays a crucial role in our analysis, since it gives rise to exponentially growing solutions whenever it takes complex values.

The values of $a$ such that $F_{\nu}(z)$ is periodic in $z$ are called the eigenvalues of the Mathieu equation. They correspond to integer values of $\nu$. They are denoted as $a_{r}(q)$ if $\nu$ is a positive integer $r$, and $b_{r}(q)$ if $\nu=-r$. It can be shown [51] that for $a>0$ one has $a_{r}>b_{r}, \nu$ is complex in the bands $b_{r}<a<a_{r}$ and real elsewhere. Moreover, for small $q$, one has $b_{r}-a_{r}=\mathcal{O}\left(q^{r}\right)$. Therefore, in the narrow resonance regime we are considering here, we will take $\nu$ complex for $b_{1}<a<a_{1}$ and $\nu$ real for $0<a \leqslant b_{1}$ and $a \geqslant a_{1}$. The series expansion in $q$ of the eigenvalues is given by

$$
a_{1}=1+q+\mathcal{O}\left(q^{2}\right) ; \quad b_{1}=1-q+\mathcal{O}\left(q^{2}\right) .
$$

If $a \neq a_{r}, b_{r}$ then $F_{\nu}(-z)$ is a solution linearly independent of $F_{\nu}(z)$. This is no longer true if $\nu$ is an integer, although an independent solution can also be constructed in that case [52]. For $\nu^{2} \neq r^{2}$, it is customary to take as independent solutions:

$$
\begin{aligned}
& c e_{\nu}(z)=\frac{1}{2}\left[F_{\nu}(z)+F_{\nu}(-z)\right] \\
& s e_{\nu}(z)=\frac{1}{2 i}\left[F_{\nu}(z)-F_{\nu}(-z)\right] .
\end{aligned}
$$

For $\nu=r, c e_{r}(z)$ are called the eigenfunctions of the Mathieu equation and so on for $\nu=-r$ and $s e_{r}(z)$. They are $2 \pi$-periodic and their $q$-expansion for $\nu^{2}=1$ is given by [52]

$$
c e_{1}(z)=\cos z-\frac{q}{8} \cos (3 z) ; \quad s e_{1}(z)=\sin z-\frac{q}{8} \sin (3 z) .
$$

Therefore, the solution $h_{1}(z, k)$ of Eq. (21) is given as a linear combination of $c e_{\nu}$ and $s e_{\nu}$ :

$$
h_{1}(z, k)=A(k) c e_{\nu}(z, k)+B(k) s e_{\nu}(z, k)
$$

where the coefficients $A(k)$ and $B(k)$ are such that the initial conditions (11) are satisfied, i.e., 


$$
\begin{aligned}
& A(k) c e_{\nu}(-\pi / 4, k)+B(k) s e_{\nu}(-\pi / 4, k)=\frac{i}{\sqrt{2 k}} \\
& A(k) \dot{c} e_{\nu}(-\pi / 4, k)+B(k) \dot{s} e_{\nu}(-\pi / 4, k)=\frac{\sqrt{2 k}}{M}
\end{aligned}
$$

where the dot means $d / d z$. Remember that $c e_{\nu}$ and $s e_{\nu}$ depend on $k$ through $a(k)$.

Even though the solutions to the Mathieu differential equation are numerically tabulated, we need their explicit form when dealing with renormalization. Such a explicit form of the solutions can be found for small $q$. Let us consider first the case when $\nu^{2} \neq r^{2}$ and real, i.e., the stable zone. Then, using the $q$-expansions given in [51,52], one has $\nu$ $=\sqrt{a}+\mathcal{O}\left(q^{2}\right)$ and the solutions are given by

$$
\begin{aligned}
& c e_{\nu}(z)=\cos (\sqrt{a} z)+\tilde{q}[\cos (\sqrt{a} z) \cos 2 z \\
& +\sqrt{a} \sin (\sqrt{a} z) \sin 2 z]+\mathcal{O}\left(q^{2}\right) \\
& s e_{\nu}(z)=\sin (\sqrt{a} z)+\tilde{q}[\sin (\sqrt{a} z) \cos 2 z \\
& -\sqrt{a} \cos (\sqrt{a} z) \sin 2 z]+\mathcal{O}\left(q^{2}\right)
\end{aligned}
$$

where

$$
\tilde{q}=\frac{a-1}{2(a-1)^{2}-q^{2}} q .
$$

If $a$ is far enough from the border points of the first band (placed at $a \simeq 1 \pm q$ ) we can simply take $\tilde{q} \simeq q /[2(a-1)]$ in Eq. (A6). The value of $a$ from which this simplification is valid can be estimated numerically, for a given $q$, by comparing to the numerical (tabulated) solutions and imposing that the difference with the approximate solution (A6) remains $\mathcal{O}\left(q^{2}\right)$.

Now, consider the unstable band, i.e., $b_{1}<a<a_{1}$. In this case, the solution (A1) reads, to leading order in $q$ [51],

$$
F_{\mu}(z)=e^{\mu z}\left[C_{1} c e_{1}(z)+S_{1} s e_{1}(z)\right]
$$

where $c e_{1}(z)$ and $s e_{1}(z)$ are given asymptotically in Eq. (A4) and the real characteristic exponent $\mu$ and the $C_{1}, S_{1}$ coefficients are given by

$$
\begin{aligned}
\mu & =\frac{1}{2} \sqrt{\left(a_{1}-a\right)\left(a-b_{1}\right)} \\
C_{1} & =\sqrt{a-b_{1}+\mu^{2}} \\
S_{1} & =\sqrt{a_{1}-a-\mu^{2}} .
\end{aligned}
$$

Note that $\mu=\mathcal{O}(q)$ and it reaches its maximum value at the band center. The dominant behavior at long times is therefore given by the positive exponentials when Eq. (A8) is replaced in Eqs. (A3) and (A5).

\section{APPENDIX B: RESULTS IN CURVED SPACE-TIME}

\section{General results}

We will collect here some of the results concerning curved space-time needed for our purposes. In this section we will consider an arbitrary metric $g_{\mu \nu}$ and in the next one we will particularize for the spatially flat RW metric. Most of the definitions used here can be found in any textbook on the subject (we are following the notation and conventions of [35]).

The covariant derivative of a contravariant vector $V^{\mu}(x)$ satisfies

$$
\sqrt{-g} V_{; \mu}^{\mu}=\partial_{\mu}\left(\sqrt{-g} V^{\mu}\right) \Rightarrow \int d^{4} x \sqrt{-g} V_{; \mu}^{\mu}=0
$$

which is the generalized Gauss theorem and $V^{\mu}(x)$ is assumed to vanish at the space-time boundary.

The Ricci tensor and scalar of curvature are defined respectively as $R_{\mu \nu}=R_{\mu \lambda \nu}^{\lambda}$ and $R=g^{\mu \nu} R_{\mu \nu}$ where the Riemann tensor is

$$
R_{\beta \gamma \delta}^{\alpha}=\partial_{\delta} \Gamma_{\beta \gamma}^{\alpha}-\partial_{\gamma} \Gamma_{\beta \delta}^{\alpha}+\Gamma_{\delta \lambda}^{\alpha} \Gamma_{\beta \gamma}^{\lambda}-\Gamma_{\gamma \lambda}^{\alpha} \Gamma_{\beta \delta}^{\lambda}
$$

and the Christoffel symbols are given in terms of the metric as

$$
\Gamma_{\mu \nu}^{\lambda}=\frac{1}{2} g^{\lambda \alpha}\left[\partial_{\mu} g_{\nu \alpha}+\partial_{\nu} g_{\mu \alpha}-\partial_{\alpha} g_{\mu \nu}\right]
$$

The classical energy-momentum tensor of the theory $T_{\mu \nu}(x)$ is defined by performing a general coordinate transformation (infinitesimal) $g^{\mu \nu}(x) \rightarrow g^{\mu \nu}(x)+\delta g^{\mu \nu}(x)$, under which the action $S=\int \sqrt{-g} \mathcal{L}$ changes as $S \rightarrow S+\delta S$ where by definition

$$
\delta S=\frac{1}{2} \int \sqrt{-g} T_{\mu \nu} \delta g^{\mu \nu} .
$$

The energy-momentum tensor thus defined is symmetric and conserved $\left(T_{\mu ; \nu}^{\nu}=0\right)$ as long as the action is a Lorenz scalar. Under the above transformation the variation of the metric determinant is given by

$$
\delta \sqrt{-g}=-\frac{1}{2} \sqrt{-g} g_{\mu \nu} \delta g^{\mu \nu}
$$

whereas the variation of the Ricci tensor yields [35]

$$
\begin{aligned}
\delta R_{\mu \nu}= & \frac{1}{2} g^{\rho \lambda}\left[\left(\delta g_{\rho \lambda}\right)_{; \mu ; \nu}-\left(\delta g_{\rho \mu}\right)_{; \nu ; \lambda}\right. \\
& \left.+\left(\delta g_{\mu \nu}\right)_{; \rho ; \lambda}-\left(\delta g_{\rho \nu}\right)_{; \mu ; \lambda}\right]
\end{aligned}
$$

which is known as the Palatini identity.

In the text we need the energy-momentum tensor defined in Eq. (47), to fourth order, i.e., when the Lagrangian is given by Eq. (16). Since we are interested only in two-point functions, we can ignore the contribution of $\mathcal{L}_{4}(U, g)$ and concentrate only in the $L_{11}$ and $L_{12}$ pieces. Therefore, using Eq. (B4), 


$$
T_{\mu \nu}^{(4,2)}(x)=-g_{\mu \nu}(x) \mathcal{L}^{(4,2)}(x)+2 \frac{\delta \mathcal{L}^{(4,2)}(x)}{\delta g^{\mu \nu}(x)}
$$

with

$$
\begin{aligned}
\mathcal{L}^{(4,2)}(x) & =-\left[L_{11} R(x) g^{\mu \nu}(x)+L_{12} R^{\mu \nu}(x)\right] F_{\mu \nu}(x) \\
F_{\mu \nu} & =\operatorname{tr}\left[\partial_{\mu} U^{\dagger}(x) \partial_{\nu} U(x)\right] \\
& =\frac{2}{f^{2}} \partial_{\mu} \tilde{\pi}^{a}(x) \partial_{\nu} \tilde{\pi}^{a}(x)+\mathcal{O}\left(\tilde{\pi}^{4}\right)
\end{aligned}
$$

in the parametrization of the $\tilde{\pi}(x)$ fields.

To calculate the variation of the Lagrangian in Eq. (B6) we use Eq. (B5) and integrate by parts taking into account Eq. (B1) as well as the properties of the covariant derivative. We find after a straightforward but lengthy calculation:

$$
\begin{aligned}
T_{\mu \nu}^{(4,2)}= & -L_{11}\left[\left(2 R_{\mu \nu}-g_{\mu \nu} R\right) g^{\alpha \beta} F_{\alpha \beta}+2 R F_{\mu \nu}\right. \\
& \left.-2 g_{\mu \nu} g^{\alpha \beta} F_{\alpha \beta ; \delta}^{; \delta}+g^{\alpha \beta}\left(F_{\alpha \beta ; \mu ; \nu}+F_{\alpha \beta ; \nu ; \mu}\right)\right] \\
& -L_{12}\left[2\left(R_{\mu}^{\alpha} F_{\nu \alpha}+R_{\nu}^{\alpha} F_{\mu \alpha}\right)-g_{\mu \nu} R^{\alpha \beta} F_{\alpha \beta}\right. \\
& \left.-g_{\mu \nu} F_{\alpha \beta}^{; \beta ; \alpha} F_{\mu \nu ; \delta}^{; \delta}+F_{\nu ; \mu ; \beta}^{\beta}+F_{\mu ; \nu ; \beta}^{\beta}\right] .
\end{aligned}
$$

The above energy-momentum tensor is symmetric, since $g_{\mu \nu}, R_{\mu \nu}$ and $F_{\mu \nu}$ are symmetric. Note that the symmetry of $F_{\mu \nu}$ is a consequence of $U$ being unitary so that

$$
\operatorname{tr}\left[\partial_{\nu} U^{\dagger} \partial_{\mu} U\right]=\operatorname{tr}\left[U \partial_{\nu} U^{\dagger} \partial_{\mu} U U^{\dagger}\right]=\operatorname{tr}\left[\partial_{\mu} U^{\dagger} \partial_{\nu} U\right] .
$$

On the other hand, in Minkowski space-time, where $R_{\mu \nu}$ $=0$ and the covariant derivatives are ordinary partial derivatives, we recover the result given in [34]. Finally, we have verified explicitly that $T_{\mu ; \nu}^{(4,2) \nu}=0$, using the equations of motion for $\mathcal{L}^{(4,2)}$, which to $\mathcal{O}\left(\tilde{\pi}^{2}\right)$ read

$$
\left\{\left[L_{11} R g^{\mu \nu}+L_{12} R^{\mu \nu}\right] \partial_{\nu} \tilde{\pi}\right\}_{; \mu}=0
$$

\section{Results for the RW conformal metric}

As explained in the text, the space-time metric we are interested in is the RW spatially flat metric in conformal time, where the scale factor is $a(t)=f(t) / f$. The line element is $d s^{2}=a^{2}(t)\left[d t^{2}-d \vec{x}^{2}\right]$ so that the elements of the metric are

$$
g_{00}(t)=a^{2}(t), \quad g_{i j}(t)=-\delta_{i j} a^{2}(t), \quad g_{i 0}=0
$$

and the metric determinant is $g \equiv \operatorname{det} g=-a^{8}(t)$. The nonvanishing Christoffel symbols for this metric are

$$
\Gamma_{00}^{0}(t)=\frac{\dot{a}(t)}{a(t)}, \quad \Gamma_{0 i}^{k}=\frac{\dot{a}(t)}{a(t)} \delta_{i}^{k}, \quad \Gamma_{i j}^{0}=\frac{\dot{a}(t)}{a(t)} \delta_{i j},
$$

and the nonvanishing elements of the Ricci tensor are given by

$$
R_{00}(t)=3\left[\frac{\ddot{a}(t)}{a(t)}-\frac{\dot{a}^{2}(t)}{a^{2}(t)}\right], \quad R_{i j}=-\left[\frac{\ddot{a}(t)}{a(t)}+\frac{\dot{a}^{2}(t)}{a^{2}(t)}\right] \delta_{i j}
$$

so that the scalar of curvature is

$$
R(t)=6 \frac{\ddot{a}(t)}{a^{3}(t)}
$$

With the above ingredients we can calculate the energymomentum tensor in Eq. (B7) for this metric. It is easy to check that $\left\langle T_{\mu \nu}^{(4,2)}\right\rangle$ is diagonal for the RW metric above, i.e., $\left\langle T_{i 0}^{(4,2)}\right\rangle=0$ and $\left\langle T_{i j}^{(4,2)}\right\rangle=0$ if $i \neq j$, as it happens also with the lowest order $\left\langle T_{\mu \nu}^{(2,2)}\right\rangle$ (see Sec. V B 1). Therefore, we only need $T_{00}$ to calculate the total energy defined in Eq. (46) to this order. We give the result here in terms of the $\pi$ fields and the $f(t)$ function, retaining only two-field terms:

$$
\begin{aligned}
E^{(4,2)}(t)= & \int d^{3} \vec{x} a^{2}(t) T_{00}^{(4,2)} \\
= & \frac{4}{f^{3}(t)} \int d^{3} \vec{x}\left\{g_{1}(t)\left[\dot{\pi}^{a}\right]^{2}+g_{2}(t)\left[\nabla \pi^{a}\right]^{2}+g_{3}(t)\right. \\
& \times\left[\pi^{a}\right]^{2}+g_{4}(t) \pi^{a} \dot{\pi}^{a}+g_{5}(t)\left(\nabla \pi^{a}\right) \cdot\left(\nabla \dot{\pi}^{a}\right) \\
& \left.+g_{6}(t) \pi^{a} \ddot{\pi}^{a}+g_{7}(t) \dot{\pi}^{a} \ddot{\pi}^{a}\right\}
\end{aligned}
$$

where we have integrated by parts neglecting total spatial derivatives, the space-time dependence of the fields has been suppressed for simplicity and

$$
\begin{aligned}
& g_{1}(t)=-3\left(2 L_{11}+L_{12}\right) \ddot{f}(t)-3\left(5 L_{11}+L_{12}\right) \frac{\dot{f}(t)^{2}}{f(t)} \\
& g_{2}(t)=\left(9 L_{11}+2 L_{12}\right) \frac{\dot{f}(t)^{2}}{f(t)}
\end{aligned}
$$$$
g_{3}(t)=-3\left(5 L_{11}+L_{12}\right) \frac{\dot{f}(t)^{4}}{f^{3}(t)}
$$$$
g_{4}(t)=3 \frac{\dot{f}(t)}{f(t)}\left[\left(2 L_{11}+L_{12}\right) \ddot{f}(t)+2\left(5 L_{11}+L_{12}\right) \frac{\dot{f}(t)^{2}}{f(t)}\right]
$$$$
g_{5}(t)=-\left(6 L_{11}+L_{12}\right) \dot{f}(t)
$$$$
g_{6}(t)=-3\left(2 L_{11}+L_{12}\right) \frac{\dot{f}(t)^{2}}{f(t)}
$$

$g_{7}(t)=3\left(2 L_{11}+L_{12}\right) \dot{f}(t)$. 
[1] D. Zschiesche et al., nucl-th/0101047 and references therein.

[2] Proceedings of Quark Matter 2001 (Elsevier, Amsterdam, in press) and references therein. Conference site: http:// www.rhic.bnl.gov/qm2001/.

[3] J. D. Bjorken, Phys. Rev. D 27, 140 (1983); J. Cleymans, R. V. Gavai, and E. Suhonen, Phys. Rep. 130, 217 (1986).

[4] M. Le Bellac, Thermal Field Theory (Cambridge University Press, Cambridge, England, 1996).

[5] A. Anselm, Phys. Lett. B 217, 169 (1989); A. Anselm and M. Ryskin, ibid. 226, 482 (1991); J. D. Bjorken, Int. J. Mod. Phys. A 7, 4189 (1992); J. P. Blaizot and A. Krzywicki, Phys. Rev. D 46, 246 (1992).

[6] K. Rajagopal and F. Wilczek, Nucl. Phys. B404, 577 (1993); S. Gavin, A. Gocksch, and R. D. Pisarski, Phys. Rev. Lett. 72, 2143 (1994).

[7] L. T. Baradzei et al., Nucl. Phys. B370, 365 (1992).

[8] T. C. Brooks et al., Phys. Rev. D 61, 032003 (2000).

[9] H. Hiro-Oka and H. Minakata, Phys. Lett. B 425, 129 (1998); 434, 461 (1998); Phys. Rev. C 61, 044903 (2000).

[10] M. Bleicher, J. Randrup, R. Snellings, and X.-N. Wang, Phys. Rev. C 62, 041901 (2000).

[11] F. Cooper, Phys. Rep. 315, 59 (1999).

[12] D. Boyanowsky, H. J. de Vega, R. Holman, and S. Prem Kumar, Phys. Rev. D 56, 5233 (1997).

[13] S. Prem Kumar, D. Boyanowsky, H. J. de Vega, and R. Holman, Phys. Rev. D 61, 065002 (2000).

[14] S. Gavin and B. Müller, Phys. Lett. B 329, 486 (1994); S. Mrowczynski and B. Müller, ibid. 363, 1 (1995).

[15] S. Weinberg, Physica A 96, 327 (1979).

[16] J. Gasser and H. Leutwyler, Ann. Phys. (N.Y.) 158, 142 (1984); Nucl. Phys. B250, 465 (1985).

[17] J. F. Donoghue, E. Golowich, and B. R. Holstein, Dynamics of the Standard Model (Cambridge University Press, Cambridge, England, 1992).

[18] A. Dobado, A. Gómez Nicola, A. López-Maroto, and J. R. Peláez, Effective Lagrangians for the Standard Model (Springer, New York, 1997).

[19] J. Gasser and H. Leutwyler, Phys. Lett. B 184, 83 (1987).

[20] P. Gerber and H. Leutwyler, Nucl. Phys. B321, 387 (1989).

[21] A. Bochkarev and J. Kapusta, Phys. Rev. D 54, 4066 (1996).

[22] D. Boyanowsky, H. J. de Vega, and R. Holman, Phys. Rev. D 51, 734 (1995).

[23] F. Cooper, Y. Kluger, E. Mottola, and J. P. Paz, Phys. Rev. D 51, 2377 (1995); F. Cooper, Y. Kluger, and E. Mottola, Phys. Rev. C 54, 3298 (1996).

[24] J. H. Traschen and R. H. Branderberger, Phys. Rev. D 42, 2491 (1990); L. Kofman, A. Linde, and A. Starobinsky, Phys. Rev. Lett. 73, 3195 (1994); Phys. Rev. D 56, 3258 (1997).
[25] J. Randrup, Phys. Rev. C 62, 064905 (2000).

[26] D. Kaiser, Phys. Rev. D 56, 706 (1997); 59, 117901 (1999).

[27] D. Boyanowsky, H. J. de Vega, R. Holman, and J. F. J. Salgado, Phys. Rev. D 54, 7570 (1996).

[28] A. Gómez Nicola and V. Galán-González, Phys. Lett. B 449, 288 (1999).

[29] D. T. Son, Phys. Rev. Lett. 84, 3771 (2000).

[30] N. D. Birell and P. C. W. Davies, Quantum Fields in Curved Space (Cambridge University Press, Cambridge, England, 1982), and references therein.

[31] J. Schwinger, J. Math. Phys. 2, 407 (1961); P. M. Bakshi and T. Mahanthappa, ibid. 4, 1 (1963); 4, 12 (1963); L. V. Keldysh, Zh. Eksp. Teor. Fiz. 47, 1515 (1964) [Sov. Phys. JETP 20, 1018 (1965)].

[32] D. Boyanowsky, D. S. Lee, and A. Singh, Phys. Rev. D 48, 800 (1993).

[33] G. Semenoff and N. Weiss, Phys. Rev. D 31, 689 (1985).

[34] J. F. Donoghue and H. Leutwyler, Z. Phys. C 52, 343 (1991).

[35] S. Weinberg, Gravitation and Cosmology (Wiley, New York, 1972).

[36] B. Lehmann-Dronke et al., Phys. Rev. D 63, 114001 (2001).

[37] D. Boyanowsky, H. J. de Vega, R. Holman, D.-S. Lee, and A. Singh, Phys. Rev. D 51, 4419 (1995).

[38] Particle Data Group, D. E. Groom et al., Eur. Phys. J. C 15, 1 (2000), available on the PDG WWW pages (URL: http:// pdg.lbl.gov/).

[39] J. A. Oller, E. Oset, and J. R. Peláez, Phys. Rev. D 59, 074001 (1999).

[40] J. Baacke, K. Heitmann, and C. Patzold, Phys. Rev. D 57, 6398 (1998).

[41] I. S. Gradshteyn and I. M. Ryzhik, Table of Integrals, Series and Products (Academic, New York, 1981).

[42] J. I. Kapusta and E. V. Shuryak, Phys. Rev. D 49, 4694 (1994).

[43] R. D. Pisarski and M. Tytgat, Phys. Rev. D 54, 2989 (1996).

[44] D. Toublan, Phys. Rev. D 56, 5629 (1997).

[45] J. M. Martínez Resco and M. A. Valle Basagoiti, Phys. Rev. D 58, 097901 (1998).

[46] J. L. Goity and H. Leutwyler, Phys. Lett. B 228, 517 (1989).

[47] A. V. Smilga, Phys. Rep. 291, 1 (1997).

[48] D. H. Rischke, Proceedings of Quark Matter 2001 (Elsevier, Amsterdam, in press) and references therein, nucl-th/0104071.

[49] C. G. Callan, S. Coleman, and R. Jackiw, Ann. Phys. (N.Y.) 59, 42 (1970).

[50] N. P. Landsman and Ch. G. van Weert, Phys. Rep. 145, 141 (1987) and references therein.

[51] N. W. Mac Lachlan, Theory and Application of Mathieu Functions (Dover, New York, 1961).

[52] M. Abramowitz and I. A. Stegun, Handbook of Mathematical Functions (Dover, New York, 1970). 